\title{
Theoretical and Experimental Study on Gear-Coupling Contact and Loads Considering Misalignment, Torque, and Friction Influences
}

\author{
Yi Guo ${ }^{\mathrm{a}, *}$, Scott Lambert ${ }^{\mathrm{a}}$, Robb Wallen ${ }^{\mathrm{a}}$, Robert Errichello ${ }^{\mathrm{b}}$, Jonathan Keller ${ }^{\mathrm{a}}$ \\ ${ }^{a}$ National Renewable Energy Laboratory \\ 15013 Denver West Parkway, Golden, CO, 80401,United States \\ ${ }^{b}$ GEARTECH \\ Townsend, Montana, United States
}

\begin{abstract}
A new analytic model addresses the tooth contact and induced loads of gear couplings that are affected by misalignment, torque, and friction. The contact model accounts for Hertzian, bending, and shear deformations of coupling teeth considering crown modifications. For a specified torque and shaft misalignment, the model calculates the number of teeth in contact, tooth load, stiffness, stress, deformation, and safety factors. The tooth load distribution around the circumference compares well with high fidelity finite-element/contact-mechanics analyses. Simulation time with the analytic model is orders of magnitude less. Using the local contact characteristics, the model computes coupling loads that are primarily caused by the disrupted tooth contact and sliding friction caused by axial motions. This analytic model was validated by experiments. The load amplitude depends on the misalignment, torque, and friction. At low torque, coupling motion was induced by the eccentricity between the hub and sleeve even with nearly perfect alignment. This eccentricity was caused by its self-weight. When torque was larger than a threshold, the motion amplitude was greatly reduced. This torque threshold was analytically derived and validated by experiments.
\end{abstract}

Keywords: Gear coupling, contact, load, misalignment, friction

${ }^{*}$ Corresponding author. Tel.: +1-303-384-7187; Fax: +1-303-384-6901.

Email address: yi.guo@nrel.gov (Yi Guo) 


\begin{tabular}{|c|c|}
\hline Nomenclature & Meaning \\
\hline$A$ & Arc of gear tooth contact \\
\hline$b$ & Backlash \\
\hline$C$ & Single pair tooth stiffness \\
\hline$E$ & Young's modulus \\
\hline$F$ & Facewidth \\
\hline$f_{c}$ & Half tooth facewidth in contact \\
\hline$f_{d}$ & Distance from the tooth center to the contact point \\
\hline$F_{c}$ & Contact length of a single tooth \\
\hline$F_{d}$ & Distance between tooth center and contact point for a tooth \\
\hline$F_{i}$ & Maximum distance between tooth center and contact point \\
\hline$i_{d}$ & Design misalignment angle \\
\hline$L_{s}$ & Coupling shaft length \\
\hline$M^{y^{\prime}}, M^{z^{\prime}}$ & Total bending moments of a coupling around $y^{\prime}$ and $z^{\prime}$ axis \\
\hline$M_{d}^{y^{\prime \prime}}, M_{d}^{z^{\prime \prime}}$ & Single tooth bending moments around $y^{\prime \prime}$ and $z^{\prime \prime}$ axis \\
\hline$M_{s}^{y^{\prime}}, M_{s}^{z^{\prime}}$ & Total bending moments of a coupling around $y^{\prime}$ and $z^{\prime}$ axis at sensors \\
\hline$M_{t}^{y^{\prime \prime}}, M_{t}^{z^{\prime \prime}}$ & Total bending moments of a coupling around $y^{\prime \prime}$ and $z^{\prime \prime}$ axis \\
\hline$N$ & Number of teeth \\
\hline$N_{c, d}$ & Number of teeth in contact at the sleeve bottom \\
\hline$N_{c, s}$ & Number of teeth in contact at the sleeve sides \\
\hline$N_{d}$ & Normal tooth load to the coupling surface \\
\hline$N_{s}$ & Number of slices along the facewidth \\
\hline$P$ & Diametral pitch \\
\hline$P_{d}$ & Individual normal tooth load \\
\hline$P_{d}^{s}$ & Load on a single tooth slice along the facewidth \\
\hline$P_{i}$ & Maximum normal tooth load \\
\hline$P_{i}^{\prime}$ & Load per unit length \\
\hline$P_{t}$ & Total mean normal tooth load \\
\hline
\end{tabular}


- continued from previous page

\begin{tabular}{|c|c|}
\hline Nomenclature & Meaning \\
\hline$P_{t}^{\prime}$ & Mean normal tooth load \\
\hline$q$ & Load sharing ratio (number of teeth in contact) \\
\hline$R$ & Pitch radius \\
\hline$R_{c}$ & Hub root crown radius at pitch diameter \\
\hline$R_{d}$ & Distance from the hub center to the contact area in the $z^{\prime}$ direction \\
\hline$R_{F}$ & Hub face crown radius (normal plane) \\
\hline$S_{c 2}$ & Design sleeve circular space width \\
\hline$S_{c 2}^{*}$ & Sleeve circular space width at the contact point \\
\hline$T$ & Transmitted torque \\
\hline$T_{m}$ & Restoring torque \\
\hline$t_{c 1}, t_{c 2}$ & Hub and sleeve tooth circular thickness \\
\hline$t_{c 1}^{*}$ & Hub tooth circular thickness at the contact point \\
\hline$W_{c}$ & Coupling weight \\
\hline$W_{d}$ & Axial load that balances a coupling tooth \\
\hline$W_{s h}$ & Coupling shaft weight \\
\hline$w_{s h}$ & Coupling shaft weight per unit length \\
\hline$Y_{1}, Y_{2}$ & Hub and sleeve tooth AGMA form factor \\
\hline$Z_{1}, Z_{2}$ & Hub and sleeve elasticity factor \\
\hline$z_{O}$ & Maximum tooth separation \\
\hline$z_{e}$ & Elastic tooth deformation \\
\hline$\alpha$ & Tooth position angle offset \\
\hline$\beta$ & Misalignment direction \\
\hline$\zeta$ & Misalignment angle \\
\hline$\xi$ & Jam angle \\
\hline$\delta_{b}$ & Tooth backlash \\
\hline$\delta_{d}$ & Normal tooth deformation \\
\hline
\end{tabular}


- continued from previous page

\begin{tabular}{|l|l|}
\hline Nomenclature & \multicolumn{1}{c|}{ Meaning } \\
\hline$\Delta_{c}$ & Accessible tooth root clearance \\
$\Delta_{r}$ & Design tooth root clearance \\
$\gamma$ & Tilt angle \\
$\Gamma$ & $\cos (\xi)$ \\
$\phi$ & Normal pressure angle \\
$\theta$ & Turning angle \\
$\mu$ & Coefficient of friction \\
$\mu_{k l}, \mu_{l}$ & Restoring function \\
\hline \hline
\end{tabular}

\section{Introduction}

Gear couplings are a common machine component used extensively in a wide range of applications including aerospace, manufacturing, automotive, and wind power. Aside from transmitting the driving torque, crowned-tooth gear couplings accommodate some axial motion and angular misalignment between the two connecting shafts. An important aspect of gear couplings is the existence of forces and moments that are transmitted to the driveline when misaligned. Amplitudes of these loads have been reported to be between $12 \%$ and $16 \%$ of the drive torque for crowned- and straight-tooth couplings [1]. These forces can increase other component loads in the driveline and could lead to high vibration.

The National Wind Technology Center (NWTC) at the National Renewable Energy Laboratory (NREL) operates two multimegawatt wind-energy-specific dynamometers. These dynamometers offer a unique opportunity to perform measurements of coupling performance. Gear couplings used in the NWTC's 5-MW dynamometer testing facility are key components that accommodate angular misalignment and transmit torque from the dynamometer gearbox to the device under test during operation. Coupling-induced loads 
are ultimately reacted by both the device under test and the dynamometer gearbox, contributing to the uncertainty in the loads applied to the wind turbine drivetrain. To better understand the coupling-induced loads and ensure safe operation of the machinery, this work investigated the performance and loads of gear couplings in the NWTC's 5-MW dynamometer through a combined modeling and testing approach.

Two primary mechanisms generate coupling loads, including 1) offsets of tooth contact on the opposite sides of the coupling and 2) sliding friction caused by tooth axial motions. Depending on the instantaneous torque and misalignment, the number of teeth in contact and contact pattern of gear couplings vary significantly. Understanding these mechanisms requires the knowledge of the local contact characteristics at individual teeth. However, studies on coupling-induced loads did not include a tooth-contact model. Different from aforementioned approaches in calculating the coupling loads, the new model described in this paper is anticipated to yield quick and accurate results by efficiently addressing the local contact characteristics. The developed model has been validated by experiment conducted at the 5-MW dynamometer.

Early coupling load models made assumptions about the number of teeth in contact and contact pattern of gear couplings. Crease [1] analytically derived the induced loads of straight-tooth couplings and assumed only two teeth in contact. Gibbons [2] presented moment and force equations for straight-tooth, diaphragm, and annular-disk couplings using an alignment load factor that is a function of misalignment angle, assuming two teeth in contact. Elkholy and Alfares [3] provided a simplified mathematical model to estimate misalignment loads of gear couplings also based on the same assumptions. Later, Cura and Mura [4] conducted an experimental study and found that Elkholy and Alfares's model greatly overestimated the coupling loads because of these simplifications. They also presented a numerical model that calculated the number of teeth in contact based on Hertzian tooth deformation affected by misalignment and tooth stiffness. The details of tooth-contact pattern across the circumference were not given. Mancuso [5] developed analytic models to calculate coupling-induced loads. The contact points were empirically estimated as one- 
third of the tooth facewidth from the flank center. Experimental data collected from a small-scale test bench were included to compare against theoretical results. Later on, Clark [6] extended Mancuso's model [5] to consider all the teeth in contact, which was suitable for high-speed couplings. Palazzolo et al. [7] compared the modeling results using Clark's method against experimental data presented by Mancuso and found reasonable agreement at high misalignment angles. Although these coupling models have proven to be reasonably accurate in predicting loads for the studied couplings, the exclusion of a tooth-contact model has limited the applications of these models across industries that have a wide range of coupling size, torque, speed, and misalignment. Furthermore, the induced moments of misaligned couplings have components around two perpendicular axes because of the shifted tooth-contact pattern around the circumference caused by the pressure angle. These early studies have not considered the effects of pressure angle on the induced loads and, therefore, the load direction was not studied.

Existing contact models were not utilized for coupling load calculations. Contact modeling of gear couplings was first reported by Moked [8] and Renzo et al. [9]. These models investigated the contact characteristics of meshing teeth, clearance, load distribution, tooth deformation, and load capacity. Renzo et al. found the number of teeth in contact increased with the decreasing misalignment and has presented an approximated computation of the number of teeth in contact at different misalignment angles. Ma and Hou [10] derived the relative displacement and tooth load distribution of a crowned gear coupling at different misalignments. Alfares et al. [11] developed a computational contact algorithm to calculate the clearance distribution of misaligned gear couplings, and results were correlated with those in Nakashima [12] and Heinz [13]. Tjernberg [14] found that tooth-spacing errors affected the number of teeth in contact during operation. Wink and Nakandakari [15] investigated the effects of tooth-spacing errors on the number of teeth in contact and axial load factor, based on the iterative procedure recommended by the American Gear Manufacturers Association (AGMA) 927 [16] and International Standards Organization (ISO) 6336-1 [17]. Guo et al. [18] developed an analytic model to evaluate the contact characteristics of 
gear couplings that considered the effects of torque, misalignment, tooth modification, and various heat-treatment methods.

Several commercial computational packages address the contact characteristics of gear couplings. However, the various tools used for design yielded different reaction forces within the mechanical system [18]. Finite-element tools are generally regarded as accurate but at a high computational cost that limits their ability to be applied over a large design space or to problems requiring real-time execution. Hong et al. [19] developed a combined finiteelement and surface-integral contact model to calculate tooth load distributions of clearancefit gear couplings. This model considered manufacturing tooth indexing errors that commonly exist for gear couplings; however, this computational model required considerable analysis time, which made the usage less practical. Later, Hong et al. [20] established a hybrid analytical-computational model to address this problem for side-fit gear couplings. The tooth compliance caused by bending, shear, contact, and torsion was treated analytically whereas the deformation between elastic bodies was solved computationally. This approach improved computational efficiency by one order of magnitude. Although computational models and contact analysis tools exist to calculate coupling-tooth contact, the design of these components is still largely based on past experience and very little operational data are available.

The objective of this study was to address both the local contact characteristics and global-induced loads of gear couplings that can execute in a fraction of the time and thus allow comparison with real-time experimental data. Experiments conducted at the NWTC's 5-MW dynamometer test facility validated the developed coupling model. The toothcontact model was verified by correlating results with finite-element/contact-mechanics analysis. For a given torque and shaft misalignment, this analytical model quickly yielded insights into relationships between the spline design parameters and resulting tooth loads; bending, contact, and shear stresses; and safety factors considering various heat treatment methods. Using the local contact characteristics, the model then computed the coupling loads that were primarily caused by the disrupted tooth contact by misalignment and slid- 
ing friction as a result of axial motions. The analytic model also calculated the maximum misalignment that a gear coupling can accommodate and the minimum torque that was required to eliminate the initial eccentricity between the coupling sleeve and hub caused by its self-weight.

\section{NREL Dynamometer Coupling Shaft and Load Application Device}

The configuration of the dynamometer coupling-shaft assembly is shown in Figure 1. The crowned-tooth couplings at each end of the 2.8-meter-long shaft share many key design dimensions and yet differ in the allowable misalignment and backlash. Table 1 details the design parameters for each coupling. The sleeve on the driving end of the shaft has a flange that connects it to the gearbox output shaft. During operation, this sleeve rotates with the coupling shaft and any movement is strictly a result of very small deflections in the gearbox and its mounting structure. The coupling shaft is comprised of two crowned-tooth hubs bolted to the shafting. The shafting is split in the middle to accommodate a torque transducer (Figure 2). The driven sleeve is attached to the shafting inside a servo-hydraulic load-application device (LAD). In operation, the LAD can be used to apply forces and moments to the device under test to reproduce wind-loading conditions. Deflections of the device under these loads results in misalignments of the shafting and crowned-tooth couplings. The amount of coupling misalignment is therefore a function of the device stiffness and the forces applied to it.

The LAD uses hydrostatic bearings attached to hydraulic actuators to achieve nearly frictionless shaft rotation while applying forces and moments in the other five degrees of freedom on the device under test. Although the load path for the LAD is through the device under test, moments and forces generated by the crowned-tooth coupling are not in the load measurement path. The range of motion of the LAD is listed in Table 2. 


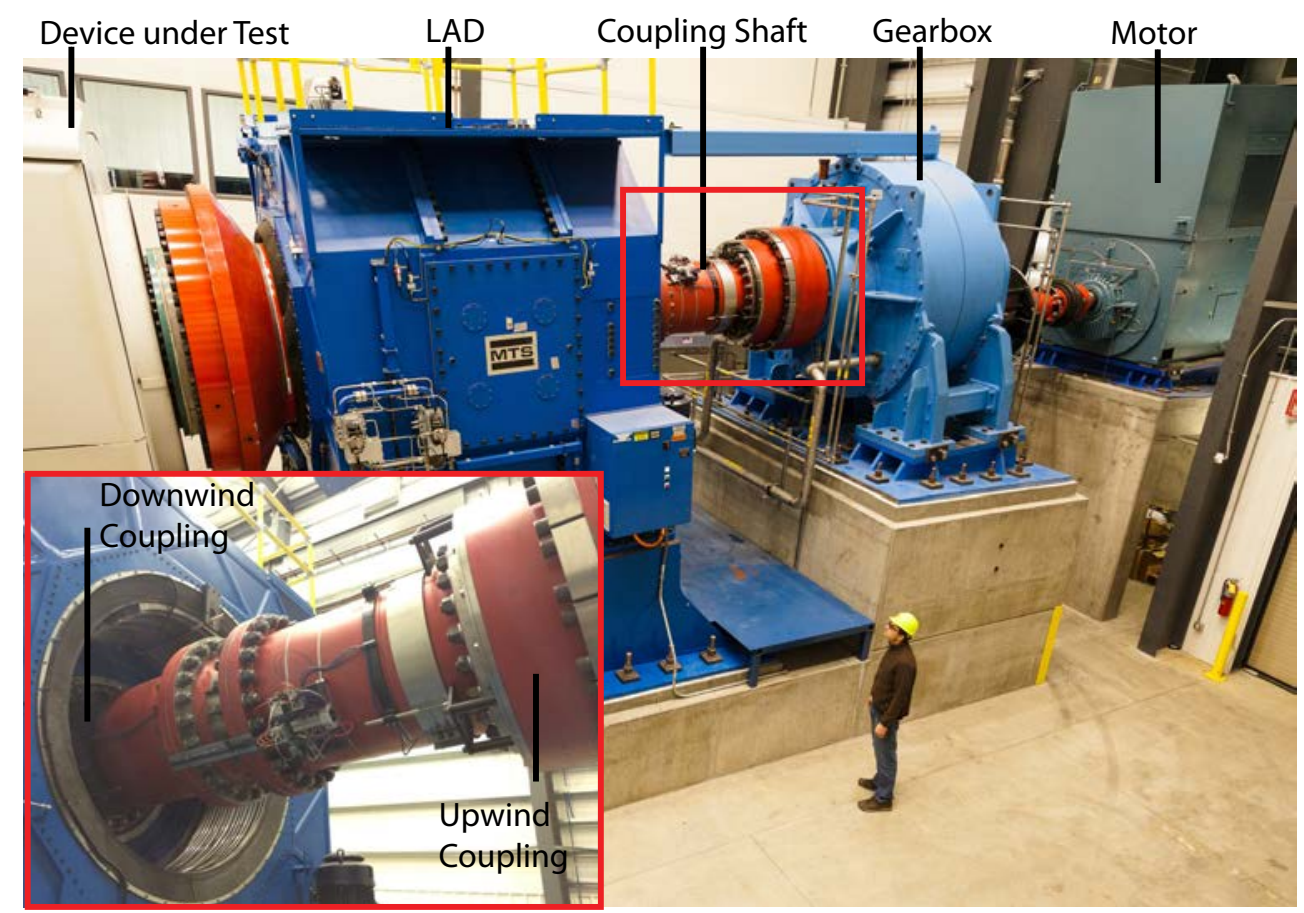

(a)

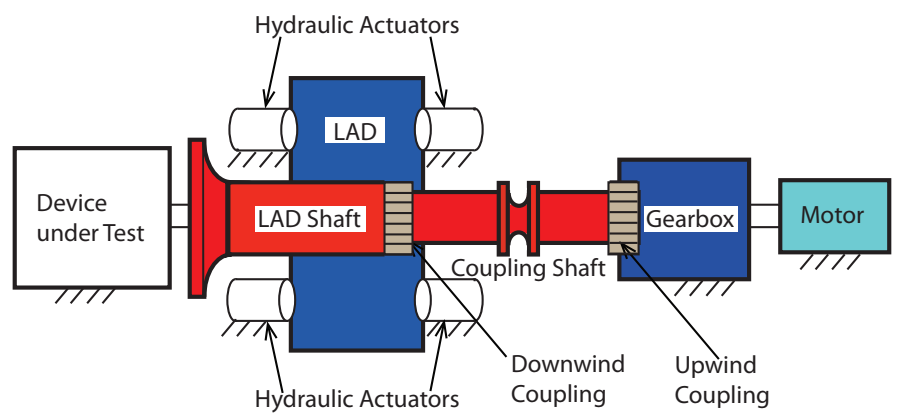

(b)

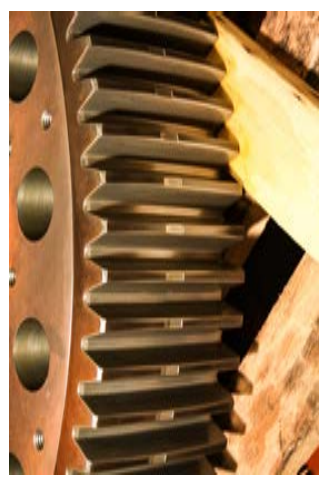

(c)

Figure 1: (a) NWTC's 5-MW dynamometer test facility, photo by Mark McDade, NREL 28218; (b) dynamometer test bench layout; and (c) coupling gear teeth, photo by Mark McDade, NREL.

\section{Experiment and Instrumentation}

The instrumentation on the shaft assembly is shown in Figure 2. Five measurements were used to compute the magnitude and direction of each of the coupling misalignments. 


\begin{tabular}{lll}
\hline Parameters & Upwind Coupling & Downwind Coupling \\
\hline \hline Number of teeth & 100 & 100 \\
Module $(1 / \mathrm{mm})$ & 10.16 & 10.16 \\
Normal pressure angle $\left(^{\mathrm{o}}\right)$ & 20.00 & 20.00 \\
Pitch diameter $(\mathrm{mm})$ & $1,016.00$ & $1,016.00$ \\
Base diameter $(\mathrm{mm})$ & 954.73 & 954.73 \\
Outer diameter $(\mathrm{mm})$ & $1,036.32$ & $1,036.32$ \\
Root diameter $(\mathrm{mm})$ & 988.77 & 987.95 \\
Center distance $(\mathrm{mm})$ & 0.00 & 0.00 \\
Circular backlash $(\mathrm{mm})$ & 0.64 & 1.97 \\
Circular tooth thickness $(\mathrm{mm})$ & 15.55 & 16.03 \\
Tool tip radius $(\mathrm{mm})$ & 4.27 & 3.18 \\
Facewidth $($ mm $)$ & 127 & 127.00 \\
Lead modification $(\mathrm{mm})$ & 0.78 at tooth edges & 1.20 at tooth edges \\
Profile tip relief $(\mathrm{mm})$ & 0.46 starting at $23.6^{\circ}$ roll angle & 0.46 starting at $23.6^{\circ}$ roll angle \\
\hline
\end{tabular}

Table 1: Specified Geometry of Gear Couplings in NWTC's 5-MW Dynamometer.

\begin{tabular}{ll}
\hline Direction & Motion Range \\
\hline \hline$x$ (axial) & $\pm 38 \mathrm{~mm}$ \\
$y$ (lateral) & $\pm 30 \mathrm{~mm}$ \\
$z$ (vertical) & $\pm 30 \mathrm{~mm}$ \\
$\theta_{y}$ (rotation about lateral) & $\pm 1.75^{\circ}$ \\
$\theta_{z}$ (rotation about vertical) & $\pm 1.75^{\circ}$ \\
\hline
\end{tabular}

Table 2: Range of Motion of the LAD.

A Heidenhain ERM 220 angular encoder provides 2,048 line counts per revolution of the shafting, resulting in an accuracy of \pm 4 minutes of shaft rotation. Four Omega linear 
variable differential transformer (LVDT) displacement transducers were used to derive the axial and angular displacement between the sleeve and hub of the couplings. The LVDT was arranged such that there are two transducers at each coupling spaced 90 degrees of rotation apart from one another. This arrangement allows for computation of shaft axial displacement from changes in the mean value of the signals. Angular misalignment was calculated using the minimum and maximum values, whereas direction can be obtained from the phase shift of the signals.

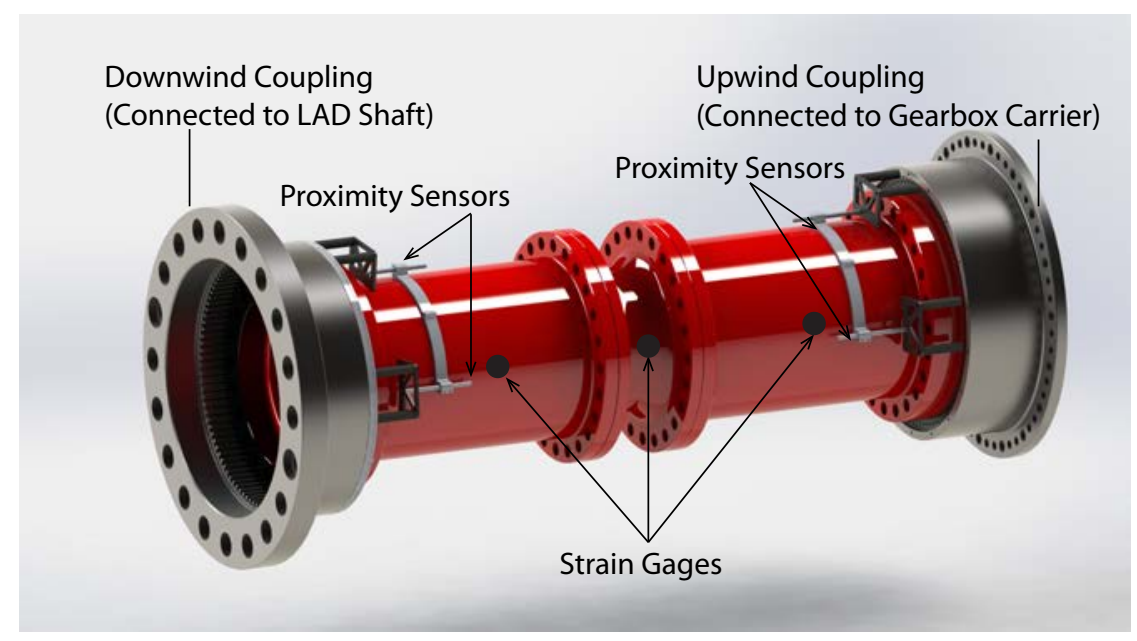

Figure 2: Instrumentation on the coupling shaft. Solidworks model by Scott Lambert, NREL

At three locations along the shaft, six sets of type-II full-bridge strain gauges were used to measure bending in three axial and two azimuthal locations along the rotating shaft assembly. The bridge configuration for the bending channels measures only bending and rejects both axial and torsional strains. Using these signals and the encoder, the bending moment in the nonrotating frame was converted from the strains in the rotating frame. As a result, a bending-moment distribution along the shaft was calculated. Prior to testing, a series of calibration forces were applied to the shaft assembly to determine the appropriate slopes and offsets for each of the bending channels. Using the bending signals and known geometry from the shaft, an estimation of friction was calculated.

Friction coefficient during operation was estimated using a combined testing and mod- 
eling approach. In [5], the moment out of plane of the misalignment can be derived as:

$$
M_{t}^{z^{\prime \prime}}=\frac{T[\sin (\varsigma)-\mu \cos (\varsigma)] \sqrt{R^{2}-\left[\frac{R}{3} \sin \left(\frac{A}{2}\right)\right]^{2}}}{R[\cos (\varsigma)-\mu \sin (\varsigma)]}
$$

The $\sqrt{R^{2}-\left[\frac{R}{3} \sin \left(\frac{A}{2}\right)\right]^{2}}$ term is dropped because it is approximately 1.0. Similarly, $\mu \sin (\varsigma)$ is approximately 0 . The final form of Eq. 1 is:

$$
M_{t}^{z^{\prime \prime}}=\frac{T[\sin (\varsigma)-\mu \cos (\varsigma)]}{R \cos (\varsigma)}
$$

where $M_{t}^{z^{\prime \prime}}$ is calculated using experimental data of shaft bending moments by:

$$
M_{t}^{z^{\prime \prime}}=\sin (\phi+\beta)\left[M_{s}^{y^{\prime}}+W_{c} \cos (\gamma) L_{s}+\frac{w_{s h} \cos (\gamma) L_{s}^{2}}{2}\right]+\cos (\phi+\beta) M_{s}^{z^{\prime}}
$$

Therefore, with measured torque, misalignment, and shaft-bending signals, the friction coefficient is estimated as:

$$
\mu=T \frac{\sin (\varsigma)}{\cos (\varsigma)}-R\left\{\sin (\phi+\beta)\left[M_{s}^{y^{\prime}}+W_{c} \cos (\gamma) L_{s}+\frac{w_{s h} \cos (\gamma) L_{s}^{2}}{2}\right]+\cos (\phi+\beta) M_{s}^{z^{\prime}}\right\}
$$

The estimated friction coefficients under various torque and misalignment conditions are listed in the Appendix. Testing at various speeds, torque, and misalignments provided data to validate the analytic model.

\section{Analytic Formulation Description}

The analytic formulation described herein includes two important aspects: contact characteristics of individual teeth and induced loads transmitted to the driveline. The toothcontact model, based on Guo et al.'s study in [18], calculates the number of teeth in contact; individual tooth load and contact area, stress, and safety factors at a given torque; and misalignment. The local contact characteristics provide crucial input for calculating the amplitude and direction of the induced loads of gear couplings. The induced loads by the coupling are primarily caused by a relocation of tooth contact by misalignment and sliding friction. Through the analytic nature of the model, solutions can be calculated two orders 
of magnitude faster than higher fidelity models, making it very useful for parametric studies during early design stages and real-time health monitoring during operations. Assumptions that the analytic formulation makes include:

- The hub and sleeve shafts are considered rigid. This assumption is reasonable because the shafts have large thicknesses to resist shaft twist. This analytic model could be conservative compared to a model that accounts for compliance of the connecting shafts.

- Only angular misalignment is considered. This assumption is valid because there was no strong evidence that the coupling teeth were moving radially and contacting the roots for the studied test articles.

- The crowning of the coupling teeth leads to constant base pitch spacing in all planes.

- The bending and contact stiffnesses are derived at a specified, steady torque, and tooth contact occurs only on the drive-side.

- The coupling teeth are equally spaced circumferentially.

- Line contact at the pitch diameter is assumed to simplify the problem.

\subsection{Local Tooth Contact}

Coupling-tooth bending stiffness is estimated using an analytic approach [21, 18], considering combined tooth deformations caused by bending and compression. The actual bending stiffness may be different because the contact is conformal between two identical involute curves. The empirical values are based on measured deformations of gear teeth, rather than gear-coupling teeth, under known test loads in which the tooth contact is localized near the pitch line. The coupling-tooth bending stiffness is:

$$
C=F E \frac{Z_{1} Z_{2}}{Z_{1}+Z_{2}}
$$

where the elasticity form factors are:

$$
Z_{1}=\frac{Y_{1}}{0.76+7.25 Y_{1}} \quad Z_{2}=\frac{Y_{2}}{0.76+7.25 Y_{2}}
$$


The total normal tooth load is:

$$
P_{t}=\frac{T}{R \cos (\phi)}
$$

where the pitch radius equals:

$$
R=\frac{N}{2 P}
$$

Without misalignment and eccentricity, all the teeth carry the same load caused by the transmitted torque and tooth-contact areas are concentrated at the tooth flank centers, as shown in Figure 3(a). When the coupling is misaligned, only a portion of the teeth are in contact with the sleeve and the tooth-load distribution changes. Some of the teeth move out of contact, as shown in Figure 3(b).

The individual normal tooth load [9] is assumed to be:

$$
P_{d}=P_{i}\left[1-\frac{z_{o}\left[\sin \left(\frac{2 \pi}{N} j+\alpha\right)\right]^{2}}{z_{e}}\right]
$$

where $z$ is the circumferential distance proportional to the turning angle $\theta$. The elastic tooth deformation equals:

$$
z_{e}=\frac{P_{i}}{C}
$$

The maximum tooth separation occurs at a turning angle of $90^{\circ}[9]$ :

$$
z_{o}=\frac{R_{c}[\tan (\zeta)]^{2}}{2 \tan (\phi)}
$$

where the hub root crown radius is:

$$
R_{c}=R_{F} \cos \left(i_{d}\right) \sin (\phi)
$$

The variable $j$ denotes the tooth number and $\alpha$ is the offset that consists of the residue of $q N$ if it is not an integer and the half tooth angle:

$$
\alpha=\frac{q N-\operatorname{int}(q N)}{4}+\frac{\pi}{N} .
$$

Load on each tooth is distributed into $N_{S}$ slices along the facewidth direction. Load on each slice is considered as:

$$
\left.P_{d}^{s}\right|_{l}=\left.P_{d} \cos \left(\frac{\pi}{N_{S}} l\right)\right|_{l=-\frac{N_{S}}{2}} ^{l=\frac{N_{S}}{2}}=\left.P_{i}\left[1-\frac{z_{0}\left[\sin \left(\frac{2 \pi}{N} j+\alpha\right)\right]^{2}}{z_{e}}\right] \cos \left(\frac{\pi}{N_{S}} l\right)\right|_{l=-\frac{N_{S}}{2}} ^{l=\frac{N_{S}}{2}} .
$$


The maximum distance between the tooth center and the farthest tooth-contact point for all teeth is estimated by its misalignment angle:

$$
F_{i}=R_{F} \sin (\zeta)
$$

Sine function distribution is assumed. The distance between the tooth center and the contact point for any individual tooth in contact is derived with the amplitude of $F_{i}$ :

$$
F_{d}=F_{i} \cos \left(\frac{2 \pi}{N} j+\alpha\right)
$$

The tooth deformation in the circumferential direction depends on tooth load as:

$$
\delta_{d}=\frac{P_{d}}{C}
$$

The width of each contact area on an individual tooth varies with tooth load:

$$
F_{c}=2 \sqrt{R_{F}^{2}-\left(R_{F}-\delta_{d}\right)^{2}}
$$

Therefore, the upper and lower boundaries of the contact area are defined as:

$$
\left[F_{d}-0.5 F_{c}, \quad F_{d}+0.5 F_{c}\right]
$$

Because of the large pressure angle of gear-coupling teeth, the tooth that carries the maximum load does not align with the misalignment axis. Instead, it deviates from the misalignment axis by the amount of pressure angle. This tooth with the maximum load aligns with the $z^{\prime \prime}$ axis, as shown in Figure 3(b).

The contact model is solved using numerical iterations and assuming initial guesses for q. Let:

$$
\begin{aligned}
& P_{t}^{\prime}=\frac{P_{t}}{N} \\
& P_{i}=\frac{P_{t}^{\prime}}{q}
\end{aligned}
$$

therefore,

$$
\frac{z_{e}}{z_{o}}=\frac{P_{i}}{C z_{o}}
$$




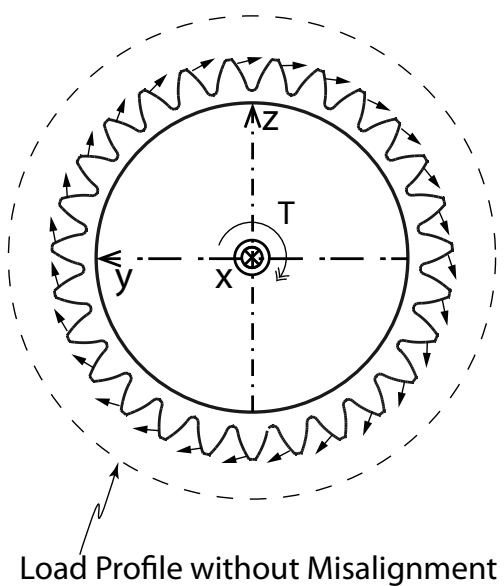

(a)

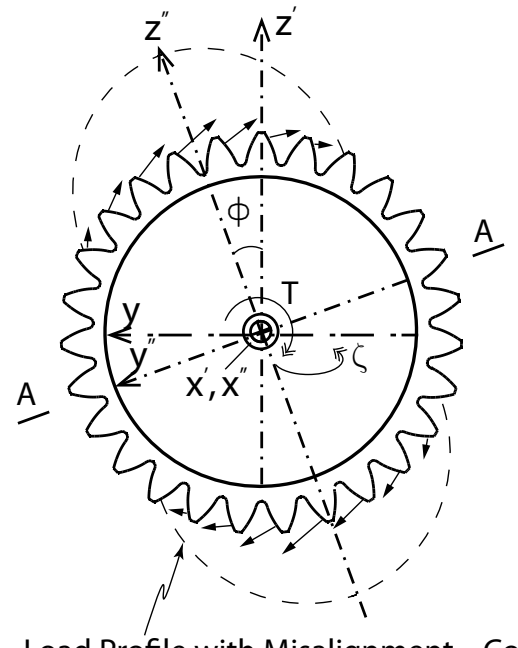

Load Profile with Misalignment

(b)

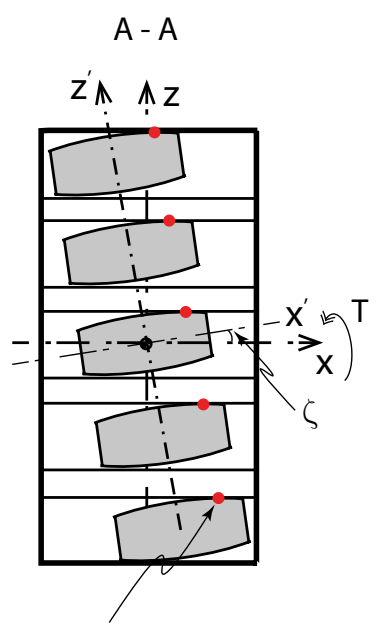

(c)

Figure 3: (a) Coupling-tooth load profile without misalignment; (b) coupling-tooth load profile with misalignment; and (c) tooth contact area with misalignment.

The new value of $q$ is calculated using the $\frac{z_{e}}{z_{o}}-q$ relation curves [9]:

$$
\left\{\begin{array}{cc}
q=0.36\left(\frac{z_{e}}{z_{o}}\right)+0.14 & 0.4 \leq \frac{z_{e}}{z_{o}}<1.0 \\
q=\sqrt{1.195+1.75\left(\frac{z_{e}}{z_{o}}\right)-\left(\frac{z_{e}}{z_{o}}\right)^{2}}-1.033 & \frac{z_{e}}{z_{o}}<0.4 \\
q=1-\frac{1}{2\left(\frac{z_{e}}{z_{o}}\right)} & \frac{z_{e}}{z_{o}} \geq 1.0
\end{array} .\right.
$$

The above process iterates until the assumed $q$ equals the calculated $q$ from Eq. 22 within the specified tolerance $\left(10^{-3}\right.$ used in this study). The calculation process is detailed in [18].

\subsection{Jam Angle}

The jam angle defines the upper boundary of the coupling-shaft misalignment. Couplings must be designed such that their jam angle is larger than the maximum misalignment expected during operations as stated in AGMA 6123-B06 [22]; however, no simple guidance is given in the standard to calculate the jam angle. Figure 4 shows the coupling tooth that contacts on both the drive- and back-side of the sleeve at the jam angle. The circumferential 
space between two adjacent teeth is limited by the amount of $S_{c 2}^{*}$, that is:

$$
2 f_{c} \sin (\xi)+\frac{t_{c 1}^{*}}{\cos (\xi)}=S_{c 2}^{*}
$$

where

$$
\begin{gathered}
f_{c}=2 R_{F} \sin \left(\frac{\xi}{2}\right) \\
t_{c 1}^{*}=t_{c 1}-2 R_{F}[1-\cos (\xi)] \\
S_{c 2}^{*}=S_{c 2}-F \sin (\xi)[\sin (\phi)]^{2}
\end{gathered}
$$

where

$$
S_{c 2}=\frac{\pi}{P}-t_{c 2}
$$

Inserting Eqs. (24) - (26) into Eq. (23) and letting $\Gamma=\cos \xi$, Eq. (23) becomes:

$$
4 \Gamma(1-\Gamma) \sqrt{\frac{1+\Gamma}{2}} R_{F}+\left\{2 R_{F}-S_{c 2}+F[\sin (\phi)]^{2} \sqrt{1-\Gamma^{2}}\right\} \Gamma+\left(t_{c 1}-2 R_{F}\right)=0 .
$$

Therefore, the jam angle equals:

$$
\xi=\cos ^{-1}\left(\Gamma_{0}\right)
$$

where $\Gamma_{o}$ is the solution of Eq. (23).

However, Eq. (28) is only valid when $f_{c} \geq \frac{F}{2}$. When $f_{c} \geq \frac{F}{2}$ :

$$
\xi=\sin ^{-1}\left(\frac{F}{2 R_{F}}\right) .
$$

The coupling could also jam in the radial direction when the relative displacement between the hub and sleeve exceeds the backlash. That is:

$$
b=2 \Delta_{c} \sin (\phi)
$$

where

$$
\Delta_{c}=\frac{1}{2} F \sin (\xi)
$$




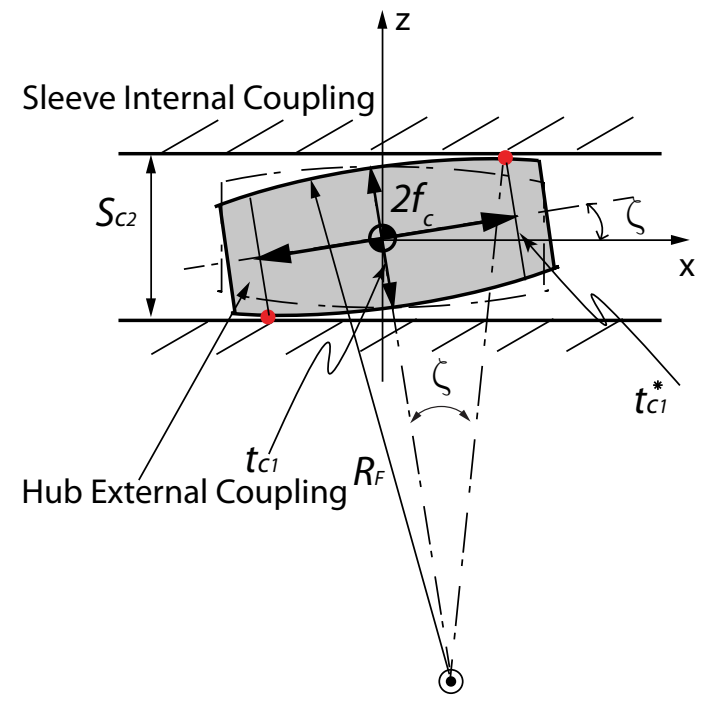

Figure 4: Misalignment spline contact on both sides of the tooth flank.

$$
b=S_{c 2}-t_{c 2}
$$

Therefore,

$$
\xi=\sin ^{-1}\left[\frac{S_{c 2}-t_{c 2}}{F \sin (\phi)}\right] .
$$

The final form of jam angle is the lowest value of all the calculated angles under all three situations:

$$
\xi=\min \left\{\cos ^{-1}\left(\Gamma_{0}\right), \sin ^{-1}\left(\frac{F}{2 R_{F}}\right), \sin ^{-1}\left[\frac{S_{c 2}-t_{c 2}}{F \sin (\phi)}\right]\right\} .
$$

The jam angle, in this formulation, is entirely determined by coupling geometric parameters. It can be enlarged primarily by adding the sleeve facewidth, increasing the coupling facewidth, decreasing hub-tooth thickness, or reducing the hub-face crown radius.

\subsection{In-Plane and Out-of-Plane Bending Moments}

The coupling stiffnesses in the radial and rotational directions are sufficiently high, such that they can be treated as rigid in this formulation; however, the coupling stiffnesses in the tilting directions are much lower to allow for the desired self-adjusting motion of the coupling shaft. When the coupling shaft tilts because of misalignment, the contact areas 
on each tooth shift away from the center of the tooth flank, as shown in Figure 3(c). The distances from the contact areas to the tooth center vary across the circumference. These offsets between tooth-contact points create bending moments on the drive shaft.

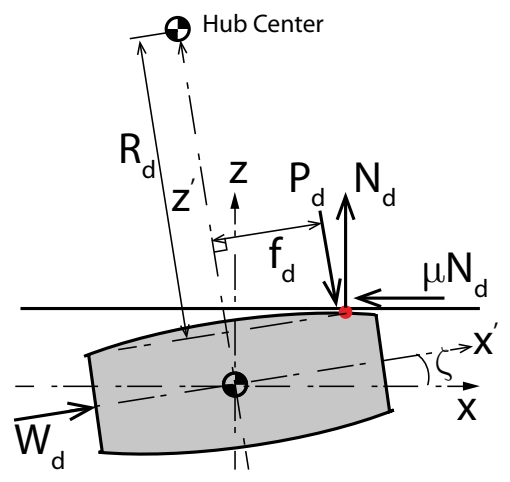

Figure 5: Force diagram of a misaligned coupling tooth.

Figure 5 shows the force diagram of the coupling at equilibrium. A force balance of the coupling in the $x^{\prime}$ and $z^{\prime}$ direction leads to:

$$
\left\{\begin{array}{l}
W_{d}-\mu N_{d} \cos (\zeta)-N_{d} \sin (\zeta)=0 \\
P_{d}+\mu N_{d} \sin (\zeta)-N_{d} \cos (\zeta)=0
\end{array}\right.
$$

Solving Eq. (36) results in:

$$
W_{d}=\frac{P_{d}[\mu \cos (\zeta)+\sin (\zeta)]}{\cos (\zeta)-\mu \sin (\zeta)} .
$$

Coupling-induced moments of a single tooth in contact around the $z^{\prime \prime}-z^{\prime \prime}$ axis equals:

$$
M_{d}^{z^{\prime \prime}}=W_{d} R_{d}
$$

where

$$
\begin{gathered}
R_{d}=\sqrt{R^{2}-f_{d}^{2}} \\
f_{d}=R_{F} \sin (\zeta) \cos \left[\left(\frac{2 \pi}{q N}\right) j+\alpha\right], \quad j=1, \cdots, q N .
\end{gathered}
$$

Coupling-induced moments of a single tooth in contact around the $y^{\prime \prime}-y^{\prime \prime}$ axis equals

$$
M_{d}^{y^{\prime \prime}}=P_{d} f_{d}
$$


Summarizing the moment vector for all teeth in contact $(1, \cdots, q N)$ and assuming the load distribution is symmetric about the misalignment axis results in:

$$
M_{t}^{y^{\prime \prime}}=4 \sum_{j=0}^{\frac{q N}{4}} P_{i} R_{F} \sin (\zeta) \cos \left(\frac{2 \pi}{N} j+\alpha\right) \cos \left(\frac{2 \pi}{q N} j+\alpha\right)\left\{1-\frac{z_{o}\left[\sin \left(\frac{2 \pi}{N} j+\alpha\right)\right]^{2}}{z_{e}}\right\}
$$

and

$$
\begin{gathered}
M_{t}^{z^{\prime \prime}}=4 \sum_{j=0}^{\frac{q N}{4}} P_{i} \cos \left(\frac{2 \pi}{N} j+\alpha\right)\left[\frac{\mu \cos (\zeta)+\sin (\zeta)}{\cos (\zeta)-\mu \sin (\zeta)}\right]\left\{1-\frac{z_{o}\left[\sin \left(\frac{2 \pi}{N} j+\alpha\right)\right]^{2}}{z_{e}}\right\} \\
\sqrt{R^{2}-\left[R_{F} \sin (\zeta) \cos \left(\frac{2 \pi}{q N} j+\alpha\right)\right]^{2}}
\end{gathered}
$$

Considering the effects of pressure angle and misalignment direction, the couplinginduced moments in the global coordinates are derived as:

$$
M^{y^{\prime}}=\cos (\phi+\beta) M_{t}^{y^{\prime \prime}}+\sin (\phi+\beta) M_{t}^{z^{\prime \prime}}
$$

and

$$
M^{z^{\prime}}=-\sin (\phi+\beta) M_{t}^{y^{\prime \prime}}+\cos (\phi+\beta) M_{t}^{z^{\prime \prime}}
$$

The final forms of Eqs. (44) and (45) become:

$$
\begin{array}{r}
M^{y^{\prime}}=4 \cos (\phi+\beta) \sum_{j=0}^{\frac{q N}{4}} P_{i} R_{F} \sin (\zeta) \cos \left(\frac{2 \pi}{N} j+\alpha\right) \cos \left(\frac{2 \pi}{q N} j+\alpha\right)\left\{1-\frac{z_{o}\left[\sin \left(\frac{2 \pi}{N} j+\alpha\right)\right]^{2}}{z_{e}}\right\}+ \\
4 \sin (\phi+\beta) \sum_{j=0}^{\frac{q N}{4}} P_{i} \cos \left(\frac{2 \pi}{N} j+\alpha\right)\left[\frac{\mu \cos (\zeta)+\sin (\zeta)}{\cos (\zeta)-\mu \sin (\zeta)}\right]\left\{1-\frac{z_{o}\left[\sin \left(\frac{2 \pi}{N} j+\alpha\right)\right]^{2}}{z_{e}}\right\} \\
\sqrt{R^{2}-\left[R_{F} \sin (\zeta) \cos \left(\frac{2 \pi}{q N} j+\alpha\right)\right]^{2}}
\end{array}
$$




$$
\begin{array}{r}
M^{z^{\prime}}=-4 \sin (\phi+\beta) \sum_{j=0}^{\frac{q N}{4}} P_{i} R_{F} \sin (\zeta) \cos \left(\frac{2 \pi}{N} j+\alpha\right) \cos \left(\frac{2 \pi}{q N} j+\alpha\right)\left\{1-\frac{z_{o}\left[\sin \frac{2 \pi}{N} j+\alpha\right]^{2}}{z_{e}}\right\}+ \\
4 \cos (\phi+\beta) \sum_{j=0}^{\frac{q N}{4}} P_{i} \cos \left(\frac{2 \pi}{N} j+\alpha\right)\left[\frac{\mu \cos (\zeta)+\sin (\zeta)}{\cos (\zeta)-\mu \sin (\zeta)}\right]\left\{1-\frac{z_{o}\left[\sin \left(\frac{2 \pi}{N} j+\alpha\right)\right]^{2}}{z_{e}}\right\} \\
\sqrt{R^{2}-\left[R_{F} \sin (\zeta) \cos \left(\frac{2 \pi}{q N} j+\alpha\right)\right]^{2}}
\end{array}
$$

The bending moments at the load-sensor locations are derived as:

$$
\left\{\begin{array}{l}
M_{s}^{y^{\prime}}=M^{y^{\prime}}-W_{c} \cos (\gamma) L_{s}-\frac{w_{s h} \cos (\gamma)}{2} L_{s}^{2} \\
M_{s}^{z^{\prime}}=M^{z^{\prime}} .
\end{array}\right.
$$

\subsection{Minimum Torque to Center the Coupling}

At very low torque, the coupling rests on the sleeve teeth because of its self-weight, which results in the eccentricity between the hub and sleeve. With this eccentricity, the coupling moves during rotation and the motion amplitude is one order of magnitude higher than that without eccentricity. With increasing torque, the lift force generated by the torque cancels out self-weight. The coupling motion diminishes after the torque exceeds a threshold. This torque threshold is the minimum restoring torque to lift the hub coupling and center it with the sleeve shaft. The restoring torque is mainly determined by the coupling geometry and can be analytically derived.

For the dynamometer low-speed shaft, the weight of the hubs and shaft must be accounted for. The sleeves are supported by the attached equipment, whereas the hub and shaft are supported by the sleeves. The potential contacting points between the hub and sleeve are illustrated in Figure 6. The actual contacting points are determined through a detailed tooth-deflection analysis.

The hub can rest at the bottom of the sleeve if there is inadequate root clearance. The allowable radial travel of the meshing teeth is linearly related to the backlash, as follows:

$$
\Delta_{c}=\frac{\delta_{b}}{2 \sin (\phi)}
$$




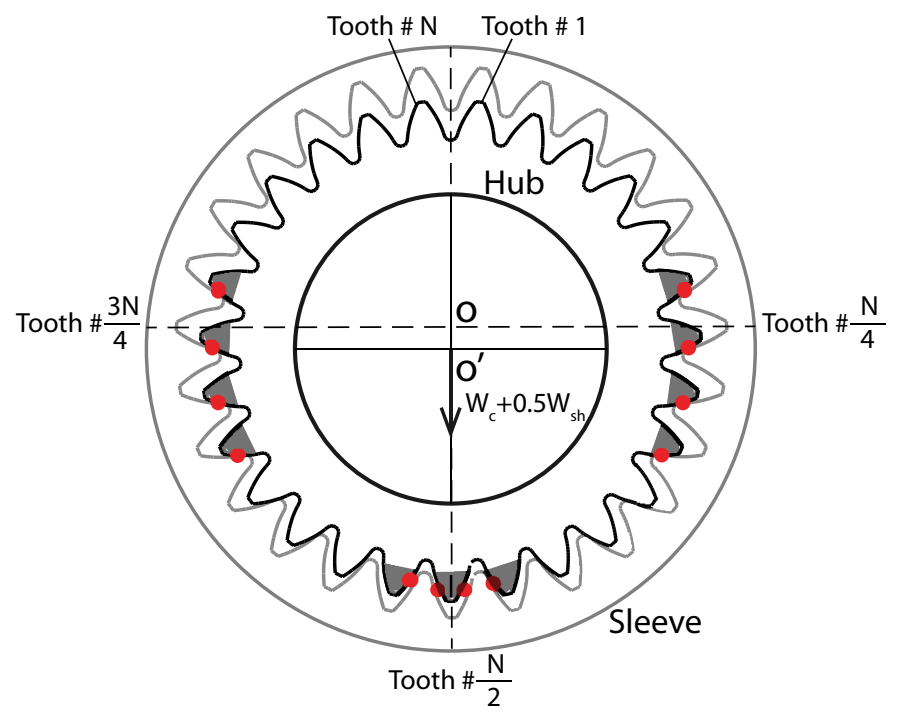

Figure 6: Coupling resting on the meshing sleeve with potential contact zones.

Under this circumstance, the restoring torque can be calculated using:

$$
T_{m}=\frac{\left(W_{c}+0.5 W_{s h}\right) R}{N_{c, b} \cos (\phi)}
$$

where $N_{c, b}=2+\sum_{k=\frac{N}{2}}^{\frac{3 N}{2}} \mu_{k}$ denotes the number of teeth in contact at the sleeve bottom. The quantity $\mu_{k}$ is the restoring function, derived as:

$$
\left\{\begin{array}{l}
\mu_{k}=1, \quad\left[\delta_{b}-\frac{\left(W_{c}+0.5 W_{s h}\right) R^{2}}{2 \cos \phi C}\right]>2 \Delta_{c} \cos \left(\frac{2 \pi k}{N}\right) \sin (\phi) \\
\mu_{k}=0, \quad\left[\delta_{b}-\frac{\left(W_{c}+0.5 W_{s h}\right) R^{2}}{2 \cos \phi C}\right] \leq 2 \Delta_{c} \cos \left(\frac{2 \pi k}{N}\right) \sin (\phi), \quad k=\frac{N}{4}, \ldots, \frac{3 N}{4} .
\end{array}\right.
$$

Typically, the coupling teeth are designed with adequate root clearance $\Delta_{c}<\Delta_{r}$ such that contacting points are on the sides of the sleeve. Under this situation:

$$
T_{m}=\frac{\left(W_{c}+0.5 W_{s h}\right) R}{N_{c, s}}
$$

where $N_{c, s}=2+2 \sum_{l=0}^{\frac{N}{2}} \mu_{l}$ denotes the number of teeth in contact at the sleeve sides. The quantity $\mu_{l}$ is the restoring function, derived as:

$$
\left\{\begin{array}{l}
\mu_{l}=1, \quad \delta_{b}\left[1-\cos \left(\frac{2 \pi}{N} l\right)\right]<\frac{\left(W_{c}+0.5 W_{s h}\right) R^{2}}{2 C} \\
\mu_{l}=0, \quad \delta_{b}\left[1-\cos \left(\frac{2 \pi}{N} l\right)\right]>\frac{\left(W_{c}+0.5 W_{s h}\right) R^{2}}{2 C}, \quad l=1, \ldots, \frac{N}{2} .
\end{array}\right.
$$




\section{Finite-Element/Contact-Mechanics Model Description}

The finite-element/contact-mechanics (FE/CM) model, Transmission3D 2.2700/0.1195, assumes conformal tooth contact throughout the entire tooth flank. The surface contact is solved by a surface integral approach that analyzes the near-field contact mechanics by integrating, in the style of a Green's function, the solution for a point load on a half space over the contact area [23, 24]. Finite-element analysis calculates far-field elastic deformations starting a small distance away from the contact area. Matching of the contact deflections and FE solutions yields a combined contact solution for near-field surface deformations. The FE/CM models of gears and bearings were validated by experiments in $[25,26]$. The FE/CM models include the flexibility of the coupling-tooth flank, rim, shaft, and tooth profile and lead modification. The FE/CM model of the upwind gear coupling is shown in Figure 7(a). Cubic elements are used to model the spline teeth and rim. The tooth-contact pattern of the coupling with a $0.5^{\circ}$ misalignment angle is illustrated. As shown in Figure 7a, the tooth-contact patterns are located at the opposite sides of the coupling and they move toward the flank edges when the coupling is misaligned. Mesh convergence has been performed to improve accuracy and results are shown in Figure 7(b). Finite elements with an average size of $25 \mathrm{~mm}$ were selected for the following analysis.

\section{Results and Discussion}

The following sections evaluate the contact characteristics and induced loads of the couplings using the developed analytic model. Because of the lack of experimental data, results of the analytic formulation about the local tooth contact were compared to these outputs from the high-fidelity FE/CM model. The coupling-induced loads were correlated with measured experimental data.

\subsection{Local Tooth Contact}

The local contact of gear couplings is primarily characterized by the tooth rotational stiffness, tooth-load distribution, and jam angle across the circumference. The rotational 


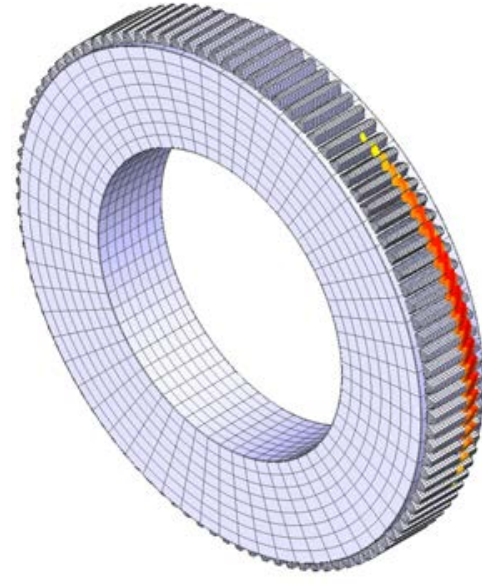

(a)

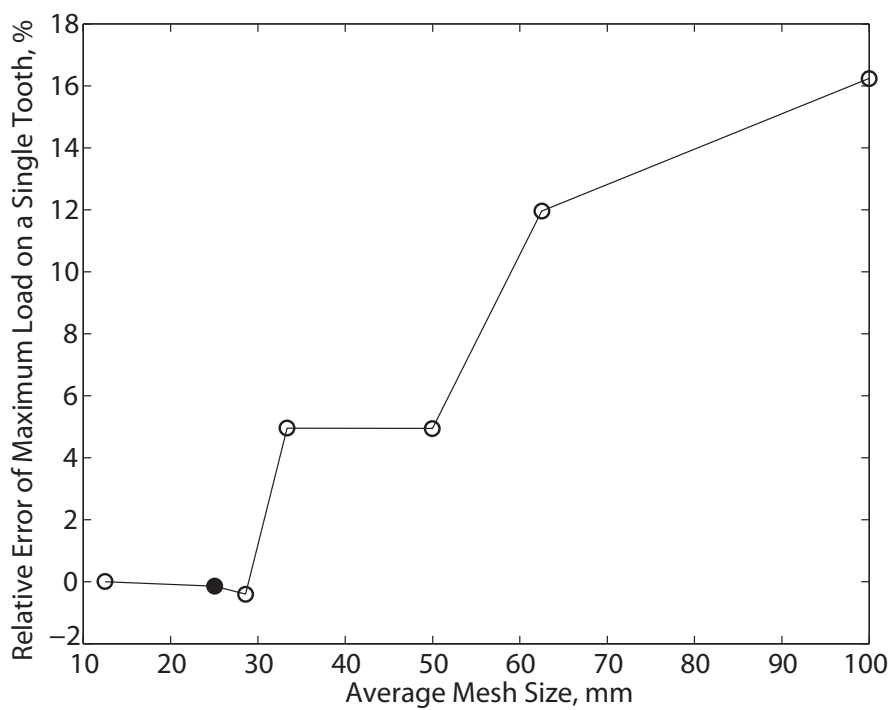

(b)

Figure 7: (a) The FE/CM model of the upwind coupling. Tooth-contact patterns with $0.5^{\circ}$ misalignment are illustrated; (b) the mesh convergence study of the finite-element model. Current mesh size is denoted by the filled circle.

stiffness of a single tooth pair determines the tooth deformation and, therefore, the number of teeth in contact at a given torque and misalignment. The rotational stiffness calculated using the analytic model was compared against the FE/CM model for a different gear coupling [18].

Figure 8 and Figure 9 show the tooth-load distributions of the upwind gear coupling at selected misalignment angles, calculated using the analytic and FE/CM models. These models are in overall good agreement, each capturing the influence of misalignment on the tooth-load distribution. The number of teeth in contact and the maximum tooth load are generally higher for the FE/CM model than for the analytic model. This is especially evident at higher misalignment angles, which is a result of the inclusion of coupling-tooth and shaft elasticity and the subsequent additional compliance in the system.

When the coupling is perfectly aligned with the sleeve shaft, all of the teeth are in contact and the torque is transmitted evenly across the teeth, as shown in Figure 8(a) and 
Figure 9(a). That is, the loads are centered on the flank and are equally distributed across all of the teeth. With misalignment, the number of teeth in contact decreases and the loads move toward the flank edges. The maximum load on a single tooth increases significantly, as shown in Figures $8(\mathrm{~b})(\mathrm{c})$ and $9(\mathrm{~b})(\mathrm{c})$. The contact pattern is symmetrically located at the opposite sides of the coupling. With a large misalignment that is close to the jam angle, less than $20 \%$ of the teeth are in contact. The contact areas are located at the flank edges and the load carried by a single tooth reaches the maximum, which is nearly four times the load without misalignment, as shown in Figures 8(d) and 9(d).

(a) Load Distribution without Misalignment, kN

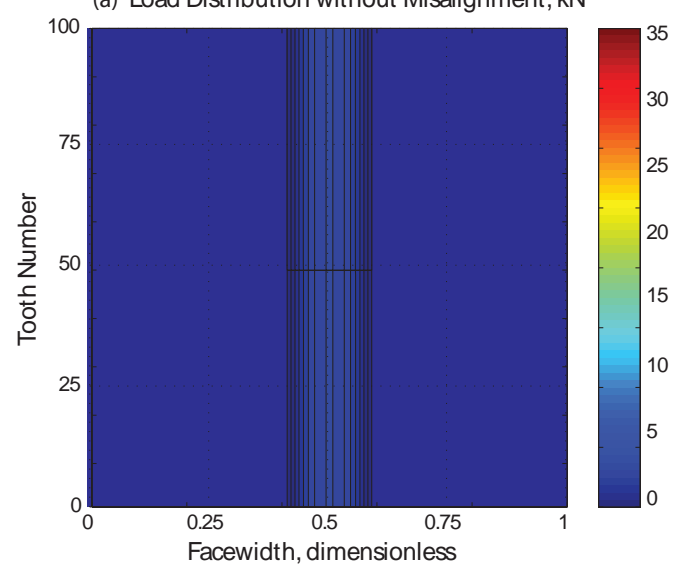

(c) Load Distribution with $0.5^{\circ}$ Misalignment, $\mathrm{kN}$

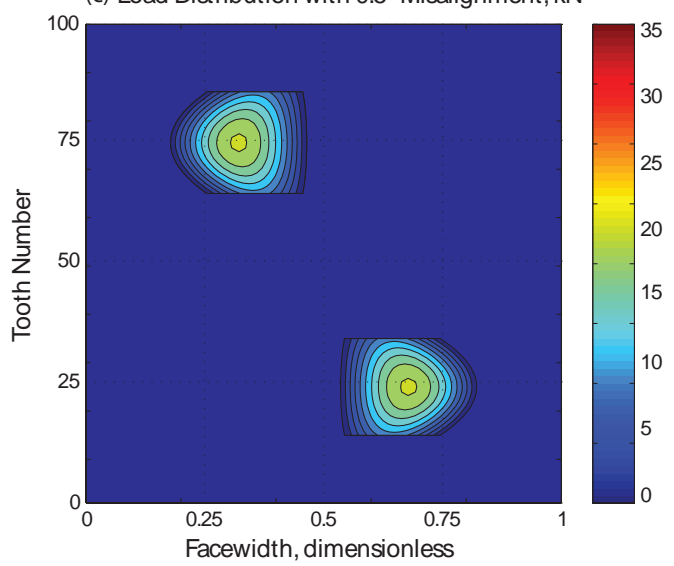

(b) Load Distribution with $0.1^{\circ}$ Misalignment, $\mathrm{kN}$

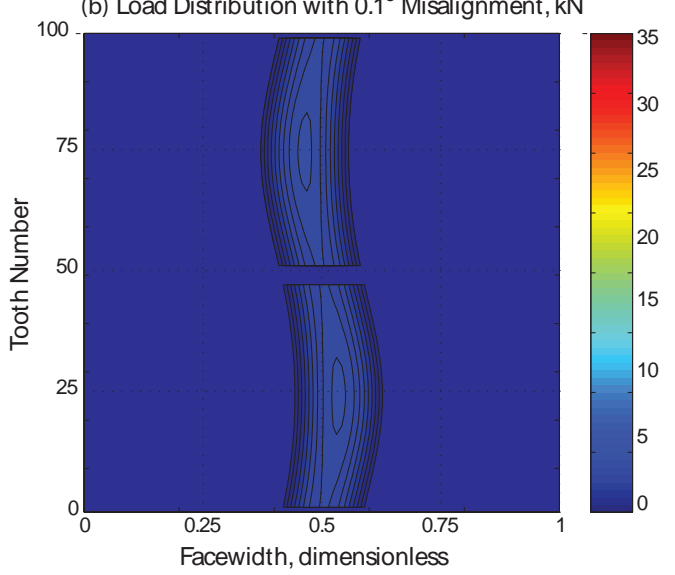

(d) Load Distribution with $0.8^{\circ}$ Misalignment, $\mathrm{kN}$

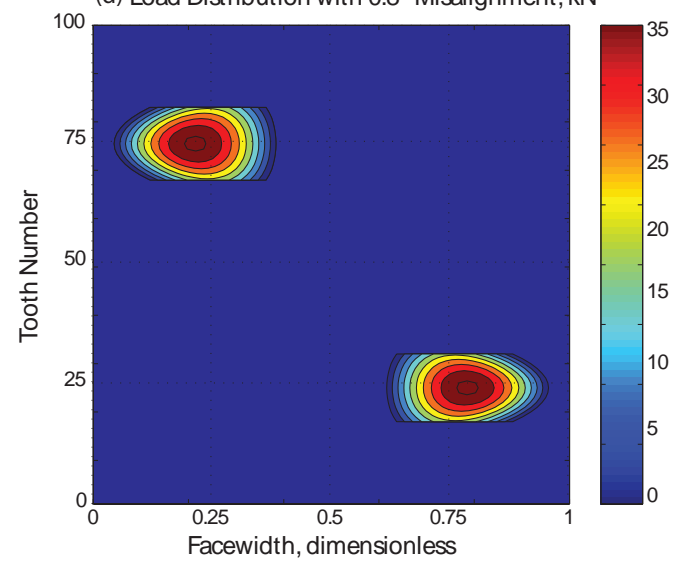

Figure 8: Upwind coupling-load distributions at (a) 0 ; (b) $0.1^{\circ}$; (c) $0.5^{\circ}$; and (d) $0.8^{\circ}$ misalignment from the analytic model with applied torque of $1.5 \mathrm{MNm}$. 
(a) Load Distribution without Misalignment, kN

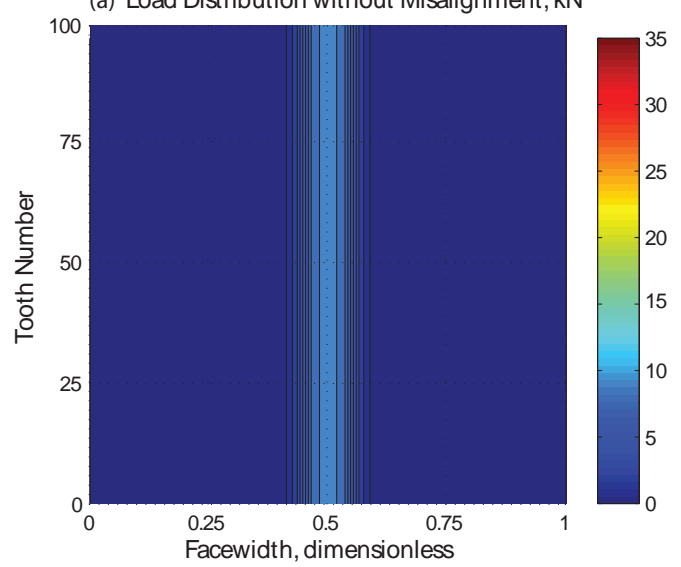

(c) Load Distribution with $0.5^{\circ}$ Misalignment, $\mathrm{kN}$

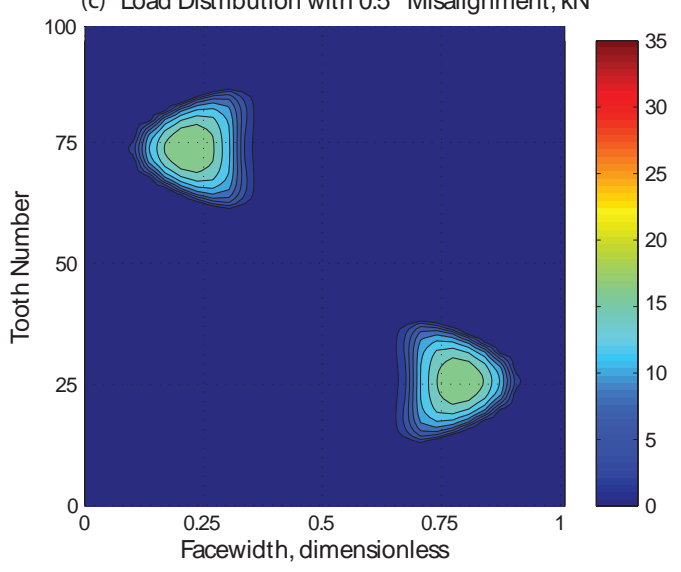

(b) Load Distribution with $0.1^{\circ}$ Misalignment, $\mathrm{kN}$

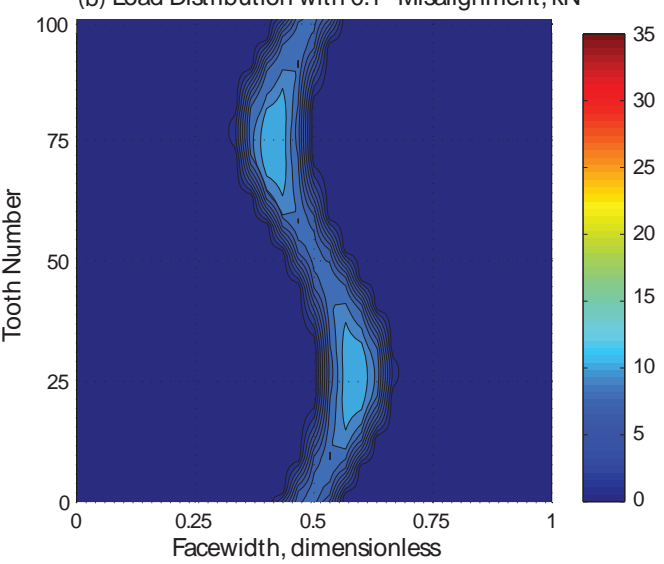

(d) Load Distribution with $0.8^{\circ}$ Misalignment, kN

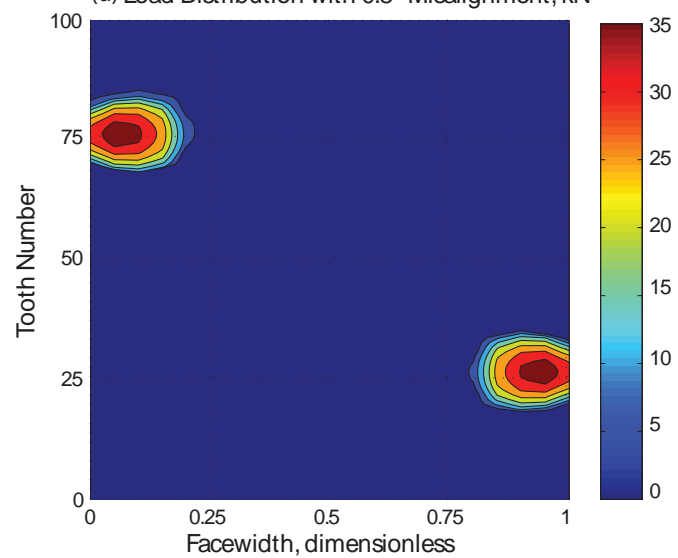

Figure 9: Upwind coupling-load distributions at (a) 0 ; (b) $0.1^{\circ}$; (c) $0.5^{\circ}$; and (d) $0.8^{\circ}$ misalignment from the FE/CM model with applied torque of $1.5 \mathrm{MNm}$.

\subsection{Jam Angle}

The calculated jam angles of the couplings using the developed analytic and FE/CM models are compared in Table 3. Reasonably good agreement is present between these two models. The analytic model estimates the jam angle is $18 \%$ lower than the FE/CM model results, which can be caused by the different tooth-profile and structural flexibilty considerations. The analytic model does not take into account the tooth profile modifications and the additional compliance in the system caused by the rim and shaft. 


\begin{tabular}{lll}
\hline Jam angle $\left(^{o}\right)$ & Upwind coupling & Downwind coupling \\
\hline \hline Analytic & 0.9 & 1.8 \\
FE/CM & 1.0 & 2.2 \\
\hline
\end{tabular}

Table 3: Jam Angles of the Upwind and Downwind Couplings

\subsection{Coupling-Induced Loads}

Combined torque and bending moments were applied to the coupling shaft through the dynamometer gearbox and LAD. The applied torque ranges from $0.1 \mathrm{MNm}$ to $2.2 \mathrm{MNm}$. The reference torque used in the paper equals $1.5 \mathrm{MNm}$, which was selected arbitrarily. The bending moments applied by the LAD to the device under test ranged from $-6.0 \mathrm{MNm}$ to $6.0 \mathrm{MNm}$ in two directions, $M_{y}$ and $M_{z}$, respectively, which resulted in coupling misalignments up to $60 \%$ of its capacity. Experiments at these combined and static loading conditions were conducted at constant operational speeds ranging from $6.0 \mathrm{rpm}$ to 15.0 rpm. The measured bending moments near the coupling were found to be almost independent of operating speed based on the data collected. Measured friction coefficients (in the Appendix) during the test were used during the load calculations. The friction coefficient is primarily affected by torque and misalignment. It increases with the torque decrease or the misalignment increase. The friction-induced loads are, therefore, significant at relatively large misalignment angles. Future experiments will investigate the effects of temperature on friction coefficients. Additionally, experimental data of the downwind coupling appear scattered at large misalignment angles, which was caused by system hysteresis of the test bench.

\subsubsection{Effects of Misalignment}

Bending moments around the $y$ and $z$ axes were applied by the LAD to misalign the coupling shaft in the $\theta_{y}$ and $\theta_{z}$ directions. Figures 10(a) and 10(b) show the misalignment amplitude and direction of the upwind and downwind couplings measured through the shaftproximity sensors. Given the applied moments around the $y$ axis ranges from $-6.0 \mathrm{MNm}$ 
to $6.0 \mathrm{MNm}$ and torque equals $1.0 \mathrm{MNm}$ and $1.2 \mathrm{MNm}$, respectively, the applied moment caused the coupling misalignment of $17 \%-60 \%$ of its capacity. The misalignment of $17 \%$ at zero applied moment suggested an initial misalignment caused by assembly tolerances and structural deflections. As shown in Figure 10(a), the misalignment amplitude linearly changes with the applied moments as anticipated. The misalignment direction, however, deviates gradually from the misalignment direction, as shown in Figure 10(b). This unexpected behavior may be caused by the compliance of the large LAD system that resulted in certain cross-coupling effects among the six degrees of freedom. Although the applied moments were only around the $y$ axis, the system responses of the coupling displacement were in multiple directions, mainly $\theta_{y}$ and $\theta_{z}$.

Figure 10(c), Figure 10(d), Figure 10(e), and Figure 10(f) compare the modeling and experimental results of $M_{s}^{y^{\prime}}$ and $M_{s}^{z^{\prime}}$ of the upwind and downwind couplings under the same loading condition as Figures 10(a) and 10(b). The measured torque, misalignment amplitude, and direction are the input of the analytic model. The time-varying friction coefficient was selected based on the instantaneous torque and misalignment at each load case (in the Appendix). Good agreement is evident between the measured and calculated $M_{s}^{y^{\prime}}$ and $M_{s}^{z^{\prime}}$ for both couplings. The analytic model overpredicts the $M_{s}^{y^{\prime}}$ of the downwind coupling at a large misalignment angle. This might be caused by the dynamic interaction of the downwind coupling with the LAD system. Further investigation is under way to understand this behavior.

Similar study was performed on the coupling loads with moments around the $z$ axis applied by the LAD system. Figures $11(\mathrm{a})$ and 11(b) show the misalignment amplitude and direction of the upwind and downwind couplings measured through the shaft-proximity sensors. The applied moment caused the coupling misalignment of $0 \%-60 \%$ of its capacity. Figure 11(c)-Figure 11(f) compare the modeling and experimental results of $M_{s}^{y^{\prime}}$ and $M_{s}^{z^{\prime}}$ of upwind and downwind couplings. Excellent agreement between the measured and calculated $M_{s}^{y^{\prime}}$ and $M_{s}^{z^{\prime}}$ is present for the upwind coupling.

As shown in Figures 10 and 11, the coupling-induced moments can reach $14 \%$ of the 

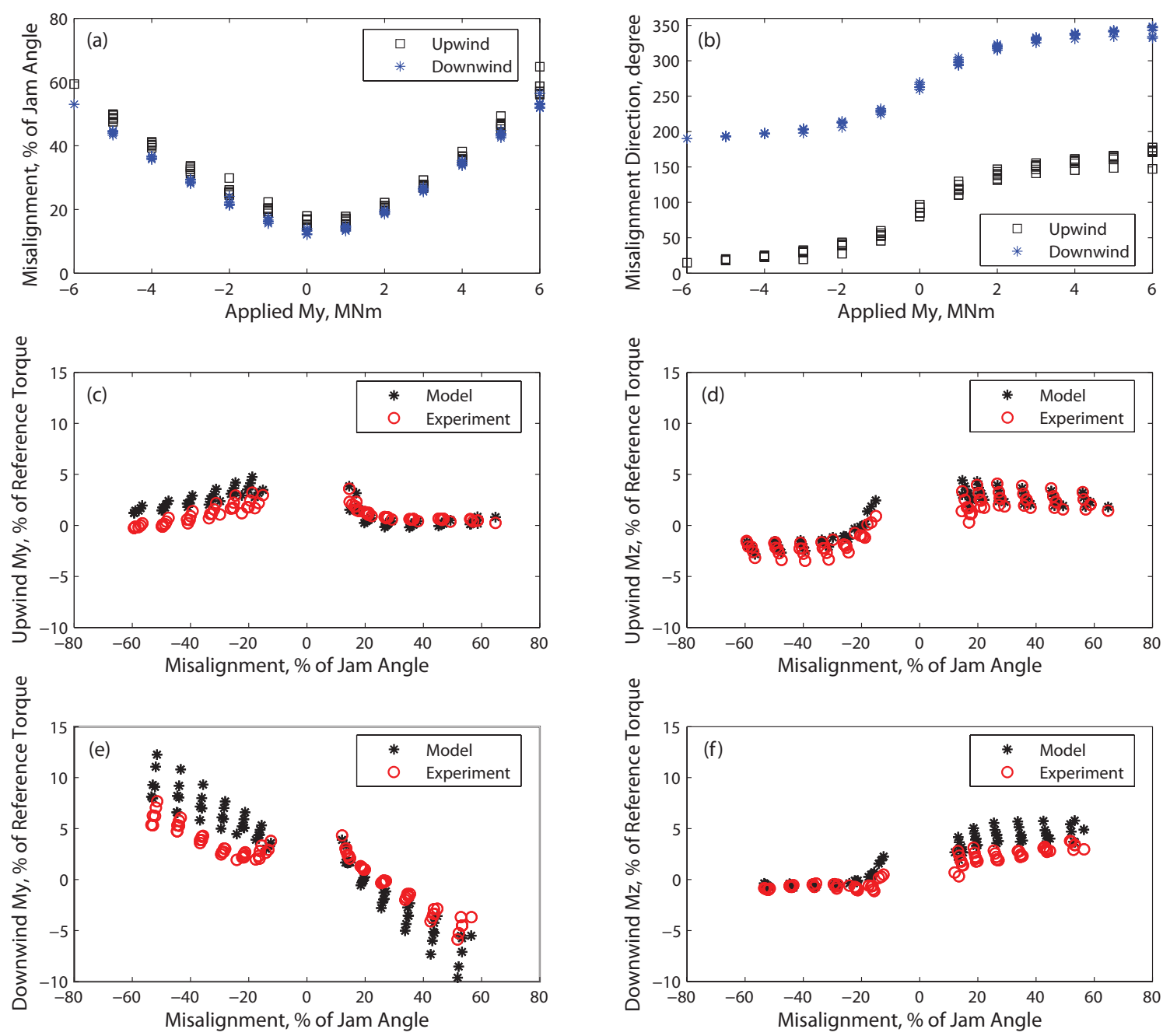

Figure 10: (a) Amplitude and (b) direction of measured coupling misalignment; upwind bending moments (c) $M_{y}$ and (d) $M_{z}$ with various measured misalignment; downwind bending moments (e) $M_{y}$ and (f) $M_{z}$ with various measured misalignment. Moments around the $y$ axis were applied to the LAD ranging from $-6.0 \mathrm{MNm}$ to 6.0 $\mathrm{MNm}$ and torque equals $1.0 \mathrm{MNm}$ and 1.2 $\mathrm{MNm}$.

reference torque of the device under test. The downwind coupling-induced loads are greater than the upwind because of its higher absolute misalignment angle. Moreover, the global moment induced by the couplings $\sqrt{\left(M_{s}^{y^{\prime}}\right)^{2}+\left(M_{s}^{z^{\prime}}\right)^{2}}$ for both couplings has a nearly linear relation with the misalignment amplitude, which agrees with the findings in $[5,7]$. 

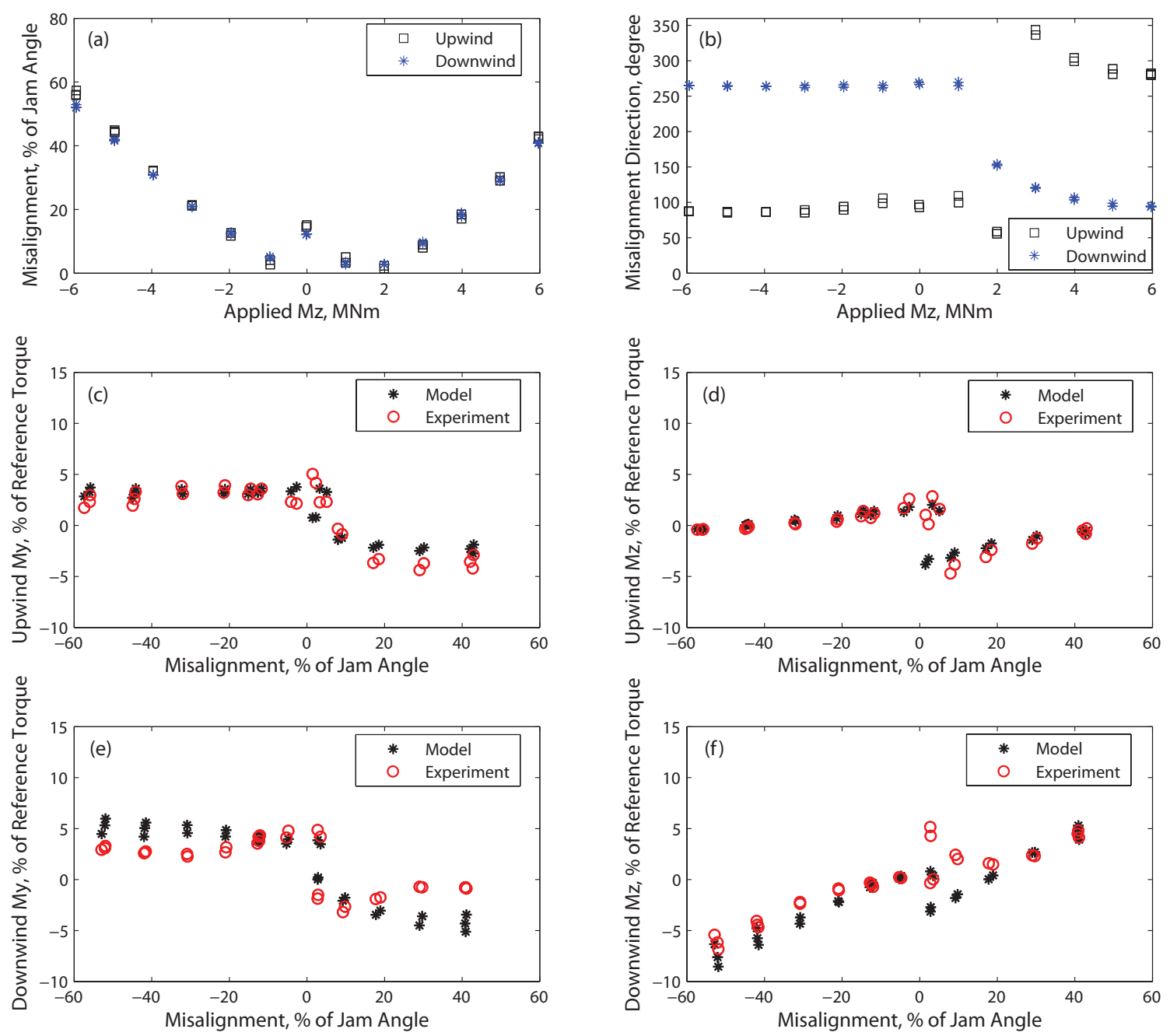

Figure 11: (a) Amplitude and (b) direction of measured coupling misalignment; Upwind bending moments (c) $M_{y}$ and (d) $M_{z}$ with various measured misalignment; downwind bending moments (e) $M_{y}$ and (f) $M_{z}$ with various measured misalignment. Moments around the $z$ were applied to LAD ranging from $-6.0 \mathrm{MNm}$ to $6.0 \mathrm{MNm}$ and torque equals $1.2 \mathrm{MNm}$.

\subsubsection{Effects of Torque}

Effects of torque on the induced loads of the downwind coupling, $M_{s}^{y^{\prime}}$ and $M_{s}^{z^{\prime}}$, are shown in Figures 12 and 13. The upwind coupling demonstrated similar behavior. Transmitted torque ranged from $10 \%$ to $95 \%$ of the reference torque of the device under test. Selected bending moments were applied by the LAD to achieve several specified misalignment angles 
that equal $\pm 30 \%, \pm 60 \%$ of their capacities in the $\theta_{y}$ and $\theta_{z}$ directions. The modeling results match the experiments well. The coupling loads increase linearly with torque. The rate of increase, however, depends on the misalignment. The load dependency on torque increases with misalignment. It is anticipated that the maximum coupling loads can exceed $14 \%$ of the reference torque at its full misalignment capacity. Additionally, for wind applications, the drivetrain torque can be much greater than the reference torque in transient events, such as start-up, emergency stop, and braking [27]. The instantaneous loads induced by the couplings, therefore, can be greater than the presented values during these dynamic operations.

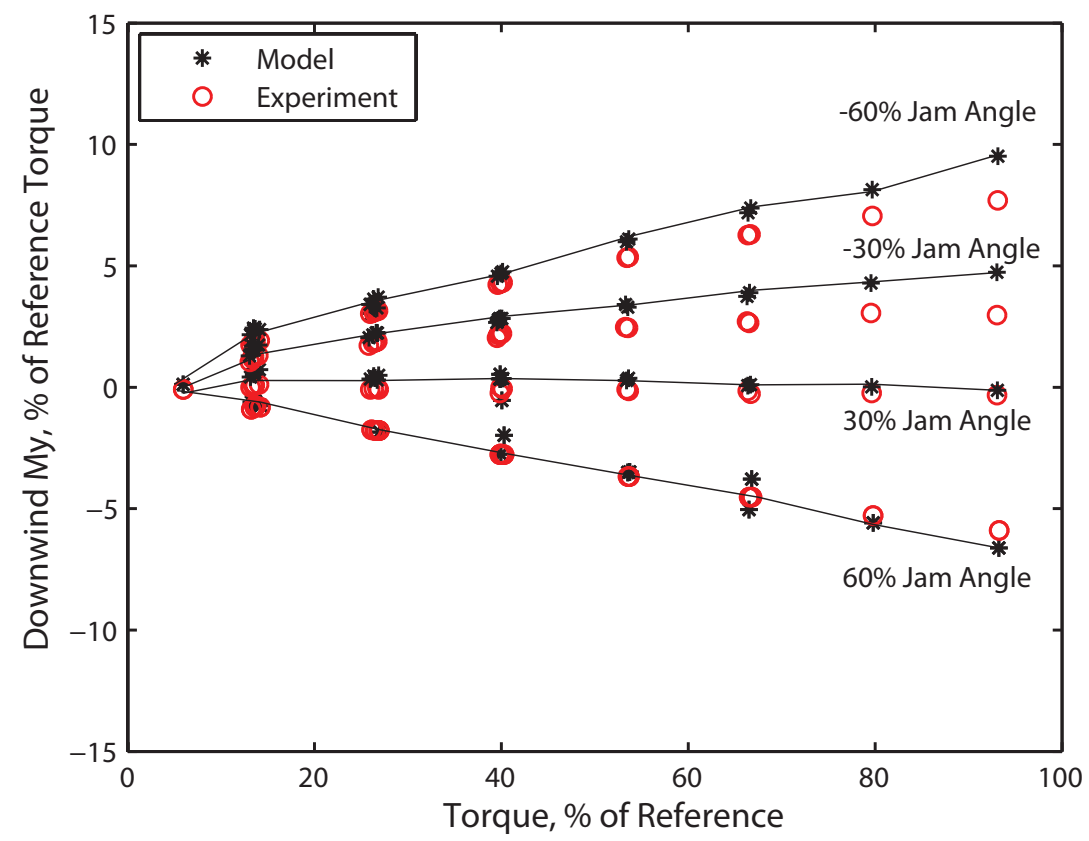

Figure 12: Downwind coupling bending moment $M_{y}$ at various torque levels.

\subsection{Effects of Restoring Torque on Coupling Motion}

A torque ramp test was conducted at the dynamometer as shown in Figure 14. Torque was increased from $0.1 \mathrm{MNm}$ to $2.2 \mathrm{MNm}$ through 17 steps. The coupling motion amplitude changed dramatically during the torque ramp, as shown in Figure 15. Significant motion of the upwind and downwind couplings between the hubs and sleeves (nearly $0.6 \mathrm{~mm}$ in 


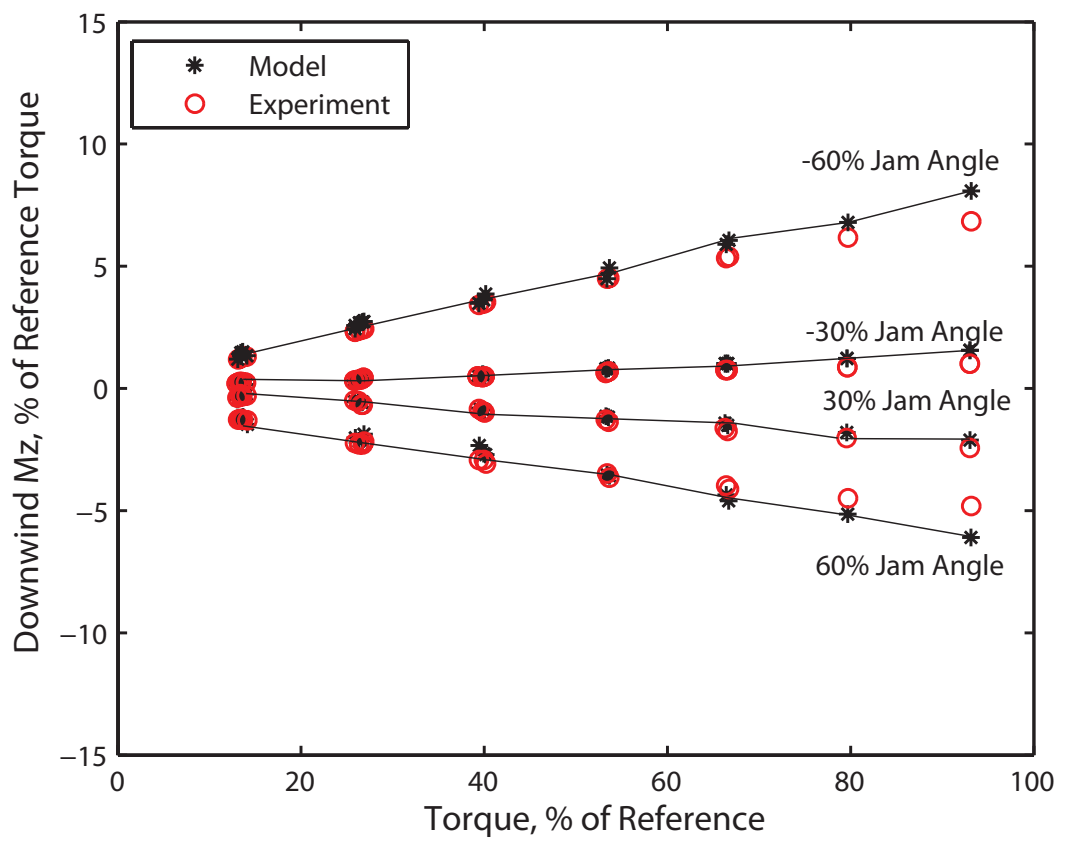

Figure 13: Downwind coupling bending moment $M_{z}$ at various torque levels.

the radial direction) occurred when torque was less than $1.2 \mathrm{MNm}(178 \mathrm{~s})$ and $1.3 \mathrm{MNm}$ (202 s), respectively, compared to higher torque levels. The coupling motion diminished when torque exceeded these two thresholds (less than $0.05 \mathrm{~mm}$ ). The coupling motion at low torque was caused by the eccentricity between the coupling hub and sleeve (Figure 6), resulting in an initial misalignment of $0.04^{\circ}-0.08^{\circ}$. When torque increased, the coupling that was initially resting on the meshing sleeve was lifted by the torque that canceled out its self-weight. Operating the coupling under low torque for a long duration could impair its health and, consequently, reduce the service life. The torque thresholds at $1.2 \mathrm{MNm}$ and 1.3 $\mathrm{MNm}$ are the restoring torques that can lift the couplings and center themselves with the sleeve. These measured restoring torques are compared against the analytic solutions derived from Eqs. (50) and (52), as shown in Table 4. The agreement between the model and experiment is reasonable with a maximum difference of $15.2 \%$ for the upwind coupling. It has been observed that the sleeve of the upwind coupling is not perfectly round because 
of manufacturing errors, which can also cause tooth spacing errors across the circumference. The analytic model assumes circular sleeve and equally spaced teeth, which can overpredict the restoring torque. Furthermore, the initial misalignment angles of both couplings are not zero. The analytical model assumes zero initial misalignment and thus cannot capture its influence on restoring torque.

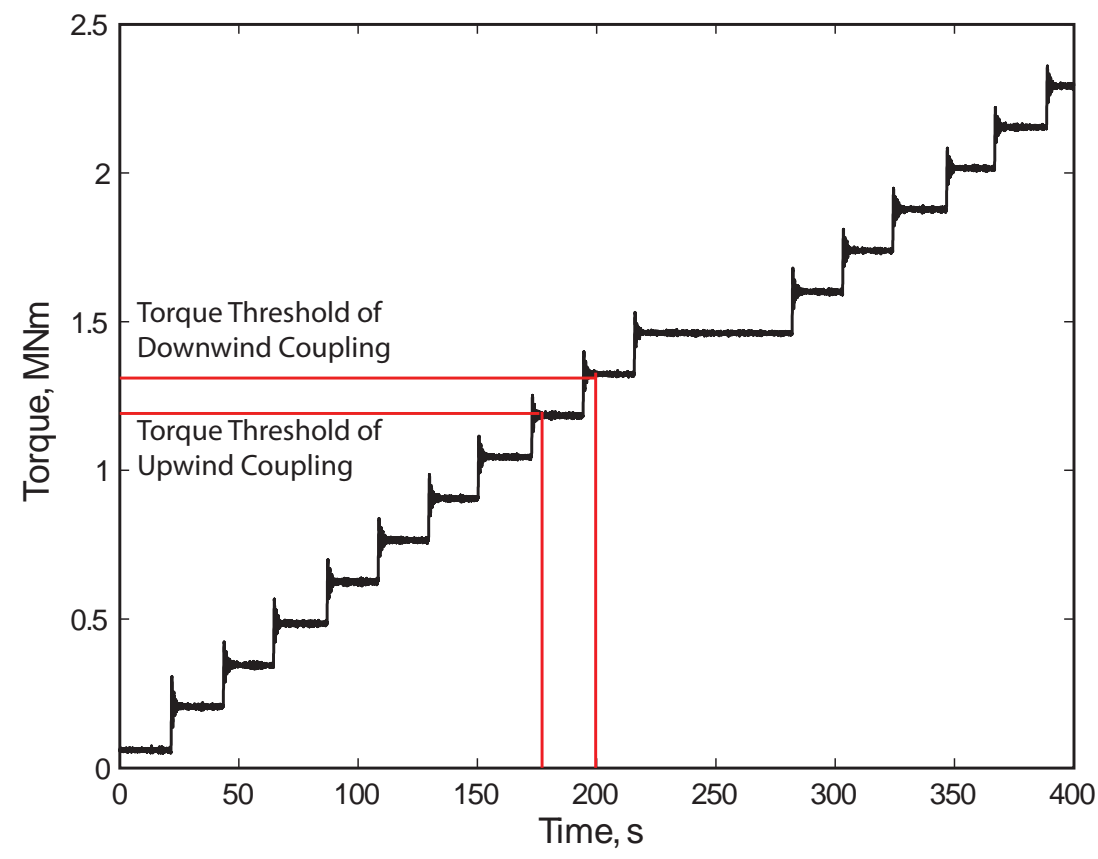

Figure 14: Torque ramp test sequence conducted at the dynamometer.

\begin{tabular}{lll}
\hline Restoring torque $(\mathrm{MNm})$ & Upwind coupling & Downwind coupling \\
\hline \hline Model & 1.36 & 1.36 \\
Experiment & 1.18 & 1.32 \\
Difference, \% & +15.25 & +3.03 \\
\hline
\end{tabular}

Table 4: Upwind and Downwind Coupling Restoring Torque. 


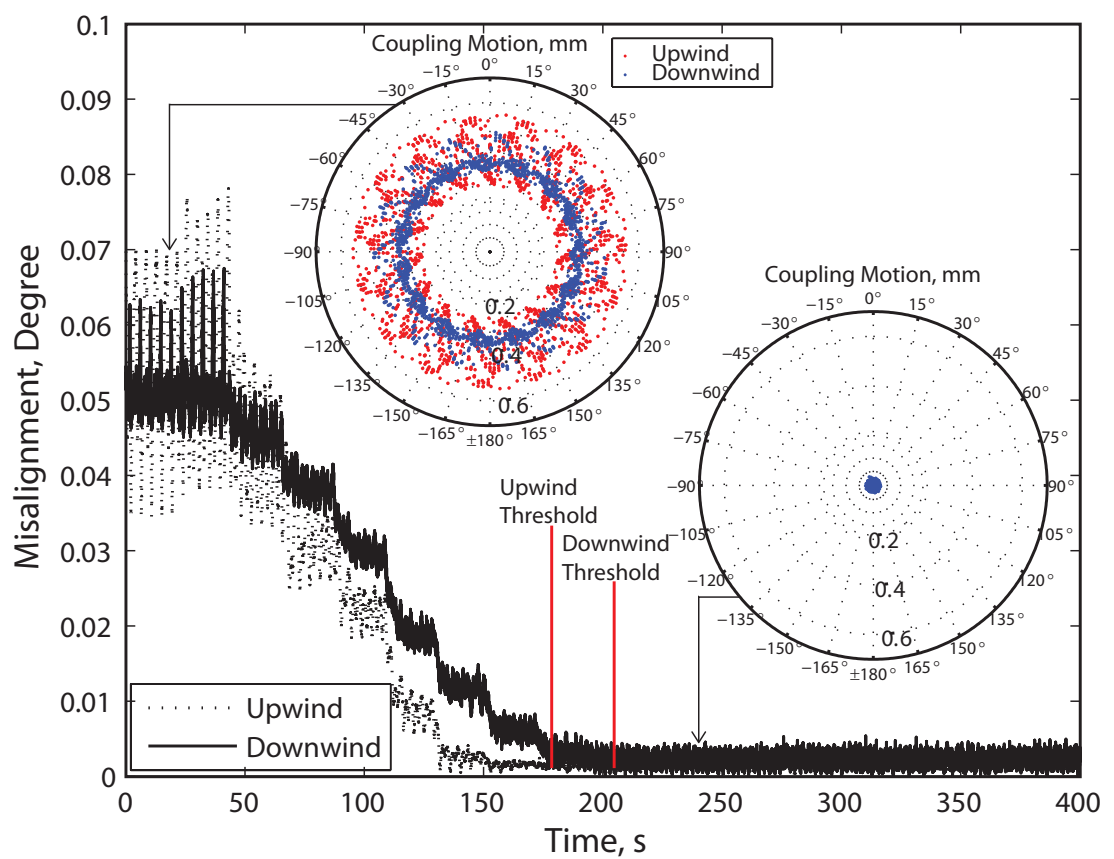

Figure 15: Upwind and downwind coupling motions and misalignment angles during the torque ramp.

\section{Conclusions}

A new analytic model for real-time design evaluation of gear couplings was developed. This model efficiently addresses both the local contact characteristics and global-induced loads of gear couplings that can be used in real-time health monitoring. Compared against previous models that calculated coupling loads, this model is more advanced by considering the local tooth contact and effects of pressure angle, which can significantly improve coupling design accuracy, reduce manufacturing cost, and decreases risks during experiments.

This model calculates the tooth-contact characteristics including number of teeth in contact, tooth-load distribution, stresses, and loads. Using the local contact characteristics, the model also predicts the induced loads that are affected by misalignment, torque, and friction. This model was validated by experiments conducted at the NWTC's 5-MW dynamometer for the coupling loads. Modeling results of tooth contact and jam angle have been correlated with FE/CM analyses.

Misalignment greatly affects the tooth-load distribution of gear couplings. When the 
coupling is aligned, torque is shared equally across all of the teeth and the load is concentrated at the tooth-flank center. When the coupling is misaligned, the number of teeth in contact decreases and the maximum load on a single tooth increases. Moreover, the contact area deviates from the tooth-flank center and moves toward the tooth edges, symmetrically located on the opposite sides of the coupling. In the extreme, the teeth are edge-loaded and at risk of failure.

Coupling-induced loads are a combined effect of misalignment, torque, and friction. The load amplitude increases nearly linearly with misalignment and torque. The load direction, however, does not align with the misalignment axis. Instead, it shifts from the misalignment axis by the pressure angle. The maximum amplitude of the coupling loads is nearly $14 \%$ of the applied torque. Sliding friction caused by the axial motions contributes to the total loads induced by reference couplings. The friction coefficient increases with the torque decrease or the misalignment increase.

Coupling can have significant motions between the hub and sleeve at low torque. This coupling motion is induced by an initial eccentricity caused by its self-weight. By increasing the transmitted torque, this motion can be greatly reduced.

\section{Acknowledgements}

This work was supported by the U.S. Department of Energy under Contract Number DE-AC36-08GO28308 with the National Renewable Energy Laboratory. Funding for the work was provided by the DOE Office of Energy Efficiency and Renewable Energy, Wind and Water Power Technologies Office.

\section{References}

[1] A. B. Crease, Force generated by gear couplings, Proceedings of the International Conference of Flexible Couplings for High Powers and Speeds (1977) B3. 1-15.

[2] C. B. Gibbons, Coupling misalignment forces, Proceedings of the Fifth Turbomachinery Symposium (1976) 111-116. 
[3] A. H. Elkholy, M. A. Alfares, Misalignment loads in splined gear couplings, International Journal of Compuational and Applied Technology 15 (1/2/3) (2002) 128-137.

[4] F. Cura, A. Mura, Experimental and theoretical investigation about reaction moments in misaligned splined couplings, Mechanical Systems and Signal Processing 45 (2014) $504-512$.

[5] J. R. Mancuso, Moments and forces imposed on power transmission system due to misalignment of a crowned tooth coupling, Master's thesis, Pennsylvania State University (1971).

[6] J. R. W. Clark, Gear coupling effects on rotordynamics, Master's thesis, Texas A\&M University (1988).

[7] A. B. Palazzolo, S. R. Locke, M. Calistrat, J. R. W. Clark, A. Ayoub, D. Calistrat, P. Tang, Gear coupling misalignment induced forces and their effects on machinery vibration, Proceedings of the Twenty-First Turbomachinery Symposium, Turbomachinery Laboratory, Texas A\&M University (1992) 83-96.

[8] I. Moked, Tooth couplings - analysis and optimization, Transactions of ASME, Series B, Journal of Engineering for Industry 90 (83) (1968) 425-434.

[9] P. C. Renzo, S. Kaufman, D. E. D. Rocker, Gear couplings, Transaction of ASME, Series B, Journal of Engineering for Industry 90 (83) (1968) 447-467.

[10] S. Ma, C. Hou, Analysis on misalignment loads of gear couplings, Transaction of ASME, Proceedings of the 1989 International Power Transmission and Gearing Conference: New Technologies for Power Transmissions of the 90's. (1989) 801-807.

[11] M. A. Alfares, A. H. Falah, A. H. Elkholy, Clearance distribution of misaligned gear coupling teeth considering crowning and geometry variations, Mechanism and Machine Theory 41 (2006) 1258-1272. 
[12] K. Nakashima, Tooth contact behavior and load distribution of gear coupling, Transaction of the Japan Society of Mechanical Engineers, Part C 54 (502) (1988) 1302-1307.

[13] R. Heinz, Untersuchung der zahnkraft- und reibungsverhaltnisse in zahnkupplungen, Konstruktion 30 (12) (1978) 483-492.

[14] A. Tjernberg, Load distribution and pitch error in a spline coupling, Materials and Design 22 (2001) 259-266.

[15] C. H. Wink, M. Nakandakari, Influence of gear loads on spline couplings, American Gear Manufacturers Association (13FTM20).

[16] AGMA, Agma 927-a01, load distribution factors - analytical methods for cylindrical gears (2000).

[17] Iso 6336-1:2006(e), calculation of load capacity of spur and helical gears - part 1: basic principles, introduction and general influence factors (annex e - analytical determination of load distribution) (1996).

[18] Y. Guo, J. Keller, R. Errichello, C. Halse, Gearbox reliability collaborative analytic formulation for the evaluation of spline couplings, National Renewable Energy Laboratory, Golden, CO Report No. TP-5000-60637.

[19] J. Hong, D. Talbot, A. Kahraman, Load distribution analysis of clearance-fit spline joint using finite elements, Mechanism and Machine Theory 74 (2014) 42-57.

[20] J. Hong, D. Talbot, A. Kahraman, A semi-analytical load distribution model for side-fit involute splines, Mechanism and Machine Theory 76 (2014) 39-55.

[21] E. Buckingham, Analytical mechanics of gears, Dover, 2011.

[22] AGMA, Agma 6123-b06: Design manual for enclosed epicyclic gear drives (2013).

[23] S. M. Vijayakar, A combined surface integral and finite element solution for a threedimensional contact problem, International Journal for Numerical Methods in Engineering 31 (1991) 524-546. 
[24] S. M. Vijayakar, H. R. Busby, L. Wilcox, Finite element analysis of three-dimensional conformal contact with friction, Computers and Structures 33 (1) (1989) 49-61.

[25] Y. Guo, T. Eritenel, T. Ericson, R. G. Parker, Vibro-acoustic propagation of gear dynamics in a gear-bearing-housing system, Journal of Sound and Vibration 333 (22) (2014) 5762-5785. doi:10.1016/j.jsv.2014.05.055.

[26] R. G. Parker, S. M. Vijayakar, T. Imajo, Non-linear dynamic response of a spur gear pair: Modelling and experimental comparisons, Journal of Sound and Vibration 237 (3) (2000) 435-455.

[27] H. Link, J. Keller, Y. Guo, B. McNiff, Gearbox reliability collaborative phase 3 gearbox 2 test plan, Technical report NREL/TP-5000-58190, National Renewable Energy Laboratory, Golden, CO (2013). 


\section{Appendix: Friction Coefficient Matrix}

\begin{tabular}{lllllllllll}
\hline Torque, $M N m$ & \multicolumn{10}{c}{ Misalignment, ${ }^{o}$} \\
\hline \hline & 0 & 0.1 & 0.2 & 0.3 & 0.4 & 0.5 & 0.7 & 0.8 & 0.9 \\
\hline 0.203 & 0.028 & 0.030 & 0.039 & 0.035 & 0.052 & 0.037 & 0.034 & 0.037 & 0.036 \\
0.400 & 0.016 & 0.018 & 0.023 & 0.016 & 0.025 & 0.031 & 0.021 & 0.020 & 0.019 \\
0.597 & 0.012 & 0.013 & 0.017 & 0.012 & 0.019 & 0.027 & 0.014 & 0.011 & 0.011 \\
0.803 & 0.009 & 0.010 & 0.013 & 0.010 & 0.014 & 0.025 & 0.014 & 0.009 & 0.009 \\
0.999 & 0.008 & 0.008 & 0.010 & 0.008 & 0.010 & 0.025 & 0.013 & 0.009 & 0.007 \\
1.196 & 0.007 & 0.007 & 0.007 & 0.006 & 0.006 & 0.025 & 0.011 & 0.007 & 0.006 \\
1.398 & 0.006 & 0.005 & 0.005 & 0.004 & 0.003 & 0.026 & 0.012 & 0.007 & 0.006 \\
\hline
\end{tabular}

Table 5: Friction coefficient of the upwind gear coupling in NWTC's 5-MW dynamometer.

\begin{tabular}{lllllllllll}
\hline Torque, $M N m$ & \multicolumn{10}{c}{ Misalignment, ${ }^{o}$} \\
\hline \hline & 0 & 0.1 & 0.2 & 0.3 & 0.4 & 0.5 & 0.7 & 0.8 & 0.9 \\
\hline 0.205 & 0.013 & 0.015 & 0.017 & 0.020 & 0.016 & 0.026 & 0.022 & 0.018 & 0.016 \\
0.397 & 0.008 & 0.009 & 0.010 & 0.013 & 0.009 & 0.015 & 0.017 & 0.012 & 0.009 \\
0.598 & 0.005 & 0.006 & 0.007 & 0.009 & 0.007 & 0.010 & 0.015 & 0.007 & 0.005 \\
0.803 & 0.004 & 0.005 & 0.006 & 0.007 & 0.005 & 0.007 & 0.014 & 0.008 & 0.004 \\
0.998 & 0.004 & 0.004 & 0.005 & 0.005 & 0.004 & 0.005 & 0.014 & 0.007 & 0.004 \\
1.196 & 0.003 & 0.004 & 0.004 & 0.004 & 0.003 & 0.003 & 0.014 & 0.006 & 0.003 \\
1.397 & 0.003 & 0.003 & 0.003 & 0.003 & 0.002 & 0.002 & 0.014 & 0.006 & 0.003 \\
\hline
\end{tabular}

Table 6: Friction coefficient of the downwind gear couplings in NWTC's 5-MW dynamometer. 